\title{
5 Kontrafaktik: Abgrenzungen, Genres, Eigenheiten
}

Nach der fiktionstheoretischen Detailerläuterung der Kontrafaktik im vorigen Kapitel soll im nachfolgenden Kapitel - metaphorisch gesprochen - ein etwas weiterer Winkel in der Betrachtung der Kontrafaktik gewählt werden. Ziele des Kapitels sind es, die Kontrafaktik von kategorial verwandten Möglichkeiten der Relationierung von Realität und Fiktion abzugrenzen, die Vorteile einer Konzeptualisierung der Kontrafaktik als genreunabhängige Referenzstruktur herauszustellen, die Genreaffinitäten kontrafaktischen Erzählens zu erläutern und schließlich mit dem Phänomen der ,Metafaktizität‘ - also der Reflexion von Wahrheit, Erfindung und Lüge innerhalb kontrafaktischer Erzählwelten - eine charakteristische Eigenheit kontrafaktischer Texte herauszuarbeiten sowie terminologisch zu fixieren.

\subsection{Realistik, Fantastik, Kontrafaktik, Faktik}

Eine bewährte Methode, sich den genauen Status eines Erzählphänomens vor Augen zu führen, besteht darin, dieses Erzählphänomen von kategorial verwandten Erzählphänomenen abzugrenzen. Zu klären ist dabei erstens, worin die jeweilige kategoriale Verwandtschaft besteht, sowie zweitens, anhand welcher Differenzkategorien sich hier sinnvolle Unterscheidungen treffen lassen. Im Folgenden soll die Kontrafaktik als eine spezielle künstlerische Möglichkeit der Relationierung von realer und fiktionaler Welt verstanden werden und in dieser Eigenschaft von drei anderen ,Weltvergleichsverhältnissen', der Realistik, Fantastik und Faktik, unterschieden werden. Definiert sind diese vier Weltvergleichsverhältnisse dabei durch die vier logisch möglichen Kombinationen des Kategorien Übereinstimmung/Nicht-Übereinstimmung von Elementen fiktionaler Welten mit allgemeinen Realitätsannahmen/Fakten der realen Welt. Da es sich bei den nachfolgenden Überlegungen zu real-fiktionalen Weltverhältnissen um einen eigenständigen Theoretisierungsvorschlag handelt, die vorgeschlagenen Unterscheidungen hohe interpretatorische Relevanz besitzen und auf die vier genannten Kategorien auch im Rahmen der konkreten Textinterpretationen verschiedentlich zurückzukommen sein wird, soll die Erläuterung der Begriffe Realistik, Fantastik, Kontrafaktik und Faktik im Folgenden in gebotener Ausführlichkeit erfolgen. Tatsächlich bildet, neben der Definition und fiktionstheoretischen Erläuterung der Kontrafaktik sowie ihrer genremäßigen Ausweitung, die Erläuterung der vier genannten Begriffe die zentrale theoretische Neuerung der vorliegenden Studie. 
Die Frage nach dem Verhältnis von realer und fiktionaler Welt ist nicht nur für die Kontrafaktik von Belang, sondern stellt sich für alle künstlerischen Verfahren, die fiktionale Welten entwerfen. Eine terminologische Gegenüberstellung, die sich in diesem Zusammenhang in der Geschichte der Ästhetik und Fiktionstheorie immer wieder findet, ist diejenige zwischen Realismus und Fantastik. Gemeint ist damit in der Regel eine Differenzierung fiktionaler Texte anhand bestimmter Eigenschaften ihrer Erzählgegenstände; gefragt wird also nach Eigenschaften auf der Ebene der histoire einer Erzählung. ${ }^{387}$ Nun erlauben bekanntlich sowohl der Begriff des Realismus als auch derjenige der Fantastik ganz unterschiedliche und zum Teil widersprüchliche definitorische Bestimmungen. Realismus bezeichnet nicht nur ein gewisses Verhältnis zur Realität (Ausschluss fantastischer Elemente), sondern auch eine Reihe spezifischer Erzählverfahren (,realistische‘ Darstellungsweisen) sowie mindestens eine literaturgeschichtliche Epoche (Bürgerlicher oder Poetischer Realismus). In der Fantastikforschung wiederum finden sich neben strukturalistischen Definitionen der Fantastik wie derjenigen von Todorov ${ }^{388}$ unter anderem auch motivgeschichtliche, anthropologische, literaturpsychologische,

387 Vgl. Wünsch: Die Fantastische Literatur der Frühen Moderne (1890-1930), S. 16. Moritz Baßler hat in jüngerer Zeit einen Realismusbegriff starkgemacht, der sich nicht auf die reale Existenzmöglichkeit von Erzählgegenständen, sondern auf das literarische Darstellungsverfahren bezieht: „Realistisch erzählte Texte sind geradezu so gemacht, dass ihre Verfahren unauffällig bleiben.“ (Moritz Baßler: Deutsche Erzählprosa 1850-1950. Eine Geschichte literarischer Verfahren. Berlin 2015, S. 12) In dieser Konzeption bildet die Fantastik - verstanden vor allem als Fantasy - keinen Gegensatz zum Realismus, sondern konstituiert im Gegenteil gerade ein besonders exemplarisch realistisches Erzählverfahren. Die traditionelle Gegenüberstellung von Realismus und Fantastik wird damit hinfällig: Der Gegenpol zu (inhaltlich problemlos rezipierbaren) realistischen Text wäre dann nicht der (inhaltlich ebenfalls problemlos rezipierbare) fantastische Text, sondern ein formal verrätselter „moderne[r] Grenztext“ (Moritz Baßler: Populärer Realismus, S. 92). Vgl. ders.: Moderne und Postmoderne; ders.: Realismus - Serialität - Fantastik. Eine Standortbestimmung gegenwärtiger Epik. In: Silke Horstkotte / Leonhard Herrmann (Hg.): Poetiken der Gegenwart. Deutschsprachige Romane nach 2000. Berlin / Boston 2013, S. 31-46.

388 Der locus classicus in Todorovs Werk lautet: „Das Fantastische ist die Unschlüssigkeit, die ein Mensch empfindet, der nur die natürlichen Gesetze kennt und sich einem Ereignis gegenübersieht, das den Anschein des Übernatürlichen hat.“ (Todorov: Einführung in die fantastische Literatur, S. 34) Diese Definition bringt allerdings die Schwierigkeit mit sich, dass sich „das Phantastische auf den Grenz-Fall der Ungewissheit [reduziert]. Nur in dem auf die Dauer der hésitation (Unschlüssigkeit) beschränkten ästhetischen Eindruck, unentschlossen zwischen Erklärbarkeit und Übernatürlichem, vermag die Phantastik zu kurzfristiger, ephemerer Gestalt zu finden: Todorov war das Kunststück gelungen, einen Gegenstand zu definieren, dem er im gleichen Atemzug energisch die materiale Existenz abstritt.“ (Hans Richard Brittnacher / Clemens Ruthner: Andererseits. Oder: Drüben. Ein erster Leitfaden durch die Welten der Phantastik. In: Peter Assmann (Hg.): Andererseits: Die Phantastik. Imaginäre Welten in Kunst und Alltagskultur. Wien 2003, S. 14-22, hier S. 17). 
und systemtheoretische Beschreibungsansätze sowie dezidiert antidefinitorische und poststrukturalistische Annäherungen an das Erzählphänomen. ${ }^{389}$

Um mögliche Begriffsverwirrungen zu vermeiden, soll im gegebenen, speziell fiktionstheoretischen Argumentationszusammenhang statt von ,Realismus‘ von ,Realistik` gesprochen werden. Dieser Begriff wurde von Frank Zipfel in Abgrenzung zur ,Phantastik“ vorgeschlagen, um „,[z]wei Grundformen fiktiver Geschichten“390 voneinander zu unterscheiden. „Mit dem Begriff Realistik soll [...] der Fall bezeichnet werden, daß die Geschichte einer Erzählung mit Blick auf das jeweils gültige Wirklichkeitskonzept möglich ist.““391 Zipfels Definition der Realistik bezieht sich dabei ausschließlich auf das, was erzählt wird, und nicht auf formale Eigenschaften der Darstellung. Unter Fantastik werden demgegenüber ,alle Geschichten verstanden [...], die Elemente enthalten, die von dem in Hinblick auf die gültige Wirklichkeitskonzeption Möglichen abweichen. “392 Durch die morphologische Angleichung der beiden Begriffe Realistik und Fantastik wird betont, dass hier eine Differenzierung anhand eines einheitlichen Unterscheidungskriteriums getroffen wird, Zipfel zufolge nämlich „der Unterscheidung zwischen möglichen und nichtmöglichen Geschichten“393 (bei einer Gegenüberstellung von ,Realismus ‘ und ,Fantastik` wäre diese Differenz sehr viel weniger deutlich).

Peter Blume hat nun Zipfels Unterscheidung zwischen Realistik und Fantastik aufgegriffen und sie um die dritte Kategorie der „,kontrafaktische[n] Fiktion“3394 ergänzt, wobei er diese dritte Kategorie als einen Subtyp fantastisch-fiktionaler Texte auffasst. Laut Blume beruht ein „phantastisch-fiktionaler Diskurs [...] auf Fiktionen, denen innerhalb des Konzeptsystems der Diskursteilnehmer notwendig der Status des

389 Ein systematischer Überblick zu Theorieansätzen der Fantastikforschung findet sich bei Brittnacher / Ruthner: Andererseits, S. 19. Siehe auch Hans Richard Brittnacher / Markus May: Phantastik-Theorien. In: Dies. (Hg.): Phantastik. Ein interdisziplinäres Handbuch. Stuttgart 2013, S. 189-197.

390 Zipfel: Fiktion, Fiktivität, Fiktionalität, S. 106. Mit ,Geschichte‘ ist hier nicht die Erzählung, sondern die histoire einer fiktionalen Erzählung gemeint (ebd., S. 76-82). Mit den Begriffen ,Realistik“ und ,Phantastik‘ sollen also „zwei grundlegende Formen der Fiktivität“ (ebd., S. 106) in fiktionalen Texten voneinander abgegrenzt werden.

391 Zipfel: Fiktion, Fiktivität, Fiktionalität, S. 107.

392 Zipfel: Fiktion, Fiktivität, Fiktionalität, S. 109. Diese Definition der Fantastik geht über die bekannte Definition Todorovs insofern hinaus, als es hier nicht um die Unschlüssigkeit hinsichtlich der Realitätskompatibilität eines bestimmten Ereignisses geht, sondern um tatsächlich fantastische - also realweltlich unmögliche - Elemente innerhalb der fiktionalen Welt. Todorov schlägt solche Elemente nicht dem Fantastischen, sondern dem Wunderbaren zu. Vgl. Todorov: Einführung in die fantastische Literatur, S. 55-74.

393 Zipfel: Fiktion, Fiktivität, Fiktionalität, S. 106.

394 Blume: Fiktion und Weltwissen, S. 138-144, hier S. 142. 
Nichtseins zugeschrieben wird.“395 Man könnte auch sagen: Fantastische Texte sind solche, die Elemente enthalten, welche notwendig fiktiv sind. Den Unterbereich der Kontrafaktik innerhalb der Fantastik differenziert Blume dann - auf das Vokabular der kognitiven Semantik zurückgreifend - wie folgt:

Phantastisch-fiktionaler Diskurs im engeren Sinn beruht auf Fiktionen, die im Widerspruch zu Konzepten stehen, die innerhalb des Konzeptsystems der Diskursteilnehmer den Status allgemeiner Gesetze innehaben, kontrafaktisch-fiktionaler Diskurs beruht auf Fiktionen, die in offenem Widerspruch zu allgemein als bekannt vorauszusetzenden, jedoch nicht gesetzesmäßigen Konzepten des Konzeptsystems der Diskursteilnehmer stehen. ${ }^{396}$

Als Untergruppe fantastischer Texte zeichnen sich Blume zufolge kontrafaktische Texte dadurch aus, dass das in ihnen Dargestellte realweltlich offenkundig unzutreffend ist, wobei dieses Unzutreffende aber anders als bei „[p]hantastisch-fiktionaler Erzählliteratur im engeren Sinn“397 nicht gegen allgemeine Naturgesetzte verstößt, sondern immerhin noch mit dem, wie Blume schreibt, „Weltbild der Sprachgemeinschaft“398 in Übereinstimmung gebracht werden kann. ${ }^{399}$

Aufgrund des anders gelagerten, speziell kognitionssemantischen Erkenntnisinteresses seiner Studie entwickelt Blume keine umfassende Theorie der Kontrafaktik. Die Frage der konkreten Fehlreferenzen, wie sie der Kontrafaktik notwendigerweise zugrunde liegen, bleibt in seinem Modell ebenso unberücksichtigt wie die Möglichkeit des Einsatzes kontrafaktischen Erzählens sowohl in fiktionalen als auch in faktualen Äußerungskontexten. Gleichwohl kann Blumes Vorschlag, das Feld der fiktionalen Literatur grob in drei Bereiche aufzuteilen, aufgenommen und im Vokabular der vorliegenden Studie reformuliert sowie systematisch erweitert werden.

Es wurde bereits ausgeführt, dass sprachliche Äußerungen grundsätzlich referieren. ${ }^{400}$ Fiktionale Texte und faktuale Texte lassen sich also nicht über das Vorliegen oder die Abwesenheit von Referenz definieren, da bei sinnvollen

395 Blume: Fiktion und Weltwissen, S. 142.

396 Blume: Fiktion und Weltwissen, S. 143.

397 Blume: Fiktion und Weltwissen, S. 144.

398 Blume: Fiktion und Weltwissen, S. 143.

399 Die Entscheidung, die Kontrafaktik der Fantastik unterzuordnen, scheint mir dabei keine zwingende Konsequenz von Blumes Modell zu sein: Ebenso gut könnte man auch den (,kontrafaktischen') Widerspruch zu Konzepten des Konzeptsystems der Diskursteilnehmer als höhergeordnetes Kriterium ansetzen und den Fall von (,phantastischen') Texten, in denen diese Abweichung gesetzmäßige Konzepte betrifft, als untergeordneten Spezialfall betrachten.

400 Siehe Kapitel 4.3.5. Transfiktionale Doppelreferenz. 
sprachlichen Äußerungen Referenz immer angenommen werden muss ${ }^{401}$; nur referieren Terme wie ,Hamlet‘, ,Sherlock Holmes‘ oder ,Einhorn“ innerhalb fiktionaler Texte eben nicht auf die reale Welt, sondern auf jene fiktionale Welt, die der jeweilige fiktionale Text entwirft. ${ }^{402}$ Da nun aber fiktionale und faktuale Texte in ähnlicher Weise auf die reale oder auf eine fiktionale Welt referieren, ergeben sich hier auch Möglichkeiten des Vergleichs: Fiktionale Welten respektive Einzelelemente innerhalb dieser fiktionalen Welten lassen sich grundsätzlich mit der realen Welt vergleichen, wobei hier je nach (Erkenntnis-)Interesse ganz unterschiedliche Kriterien angelegt werden können. Im gegebenen Zusammenhang von Bedeutung ist die Frage, inwiefern die Referenzen fiktionaler Texte, falls sie auf die reale Welt bezogen würden, mit Wahrheitsannahmen über diese, die reale Welt, vereinbar wären.

Hier lassen sich nun basale Unterscheidungen nach zwei Kriterien vornehmen: Differenziert werden kann danach, ob erstens die fiktionale Welt mit der realen Welt übereinstimmt respektive übereinstimmen könnte oder nicht, und ob zweitens diese (Nicht-)Übereinstimmung sich auf allgemeine Möglichkeitsannahmen der Realität oder auf konkrete Einzelinformationen respektive Fakten bezieht. Die Differenzkategorien der ersten Unterscheidung sollen im Folgenden als Vereinbarkeit und Nicht-Vereinbarkeit bezeichnet werden, wobei mit, Vereinbarkeit' stets die Vereinbarkeit mit Wahrheitsannahmen über die reale Welt gemeint ist. Nicht-Vereinbarkeit mit der Realität impliziert dabei zugleich Fiktivität, da etwas prinzipiell Unmögliches auch nicht konkret zutreffend sein kann. ${ }^{403}$ Umgekehrt wird durch eine Vereinbarkeit mit der Realität nicht zugleich Faktizität impliziert: Die meisten realistischen Romane enthalten Elemente, die zwar möglich, aber nicht real sind, also realweltlich nicht referentialisiert werden können. Die zweite Unterscheidung lässt sich mit den Begriffen konkret und allgemein anzeigen, es wird also danach gefragt, ob die Abweichung respektive Übereinstimmung zwischen Elementen der fiktionalen Welt und solchen der realen Welt sich auf konkrete Fakten oder auf allgemeine Realitätsannahmen bezieht.

401 Literarische Texte ohne Referenzfunktion sind etwa das Lautgedicht oder bestimmte Beispiele konkreter Poesie. Allerdings kann man in diesen Fällen nicht mehr eigentlich von sprachlichen Äußerungen sprechen. Vgl. Blume: Fiktion und Weltwissen, S. $88 \mathrm{f}$.

402 Vgl. Köppe: Die Institution Fiktionalität, S. 38.

403 Zu Erzählgegenständen, die Fiktivität implizieren, bemerkt Zipfel: „Native und surrogate objects stellen die eigentlichen Fiktivitätsfaktoren von Geschichten dar: native objects natürlich deshalb, weil sie per definitionem Objekte sind, die nur in der Geschichte und nicht in der Wirklichkeit vorkommen; surrogate objects deshalb, weil sie gegenüber ihren Entsprechungen in der realen Welt in einer Art und Weise verändert dargestellt werden, daß man sie ebenfalls als fiktive Objekte ansehen kann und muß.“ (Zipfel: Fiktion, Fiktivität, Fiktionalität, S. 100 f.). 
Die Differenzierung zwischen konkreten Fakten der Enzyklopädie auf der einen und allgemeinen Realitätsannahmen auf der anderen Seite lässt sich zwar nicht in jedem Einzelfall trennscharf durchhalten. ${ }^{404}$ Als Strukturierungsvorschlag für verschiedene Aspekte des Weltwissens erscheint diese Trennung aber nichtsdestoweniger sinnvoll, da sie, wie im Folgenden gezeigt werden soll, interpretatorisch durchaus relevant ist und von den meisten Lesern intuitiv ohnehin vorgenommen wird. ${ }^{405}$ Im Einzelfall bereitet die Entscheidung darüber, auf welchen Bereich des Weltwissens - allgemeine Realitätsannahmen oder konkretes Faktenwissen - sich ein fiktionales Element bezieht, nur selten Probleme. Im Rahmen der Interpretationspraxis kann hier letztlich pragmatisch verfahren werden; anstatt also präzise Kriterien der Differenzierung zwischen allgemeinen Realitätsannahmen und konkreten Fakten anzuführen, kann hier - wie überhaupt bei der Interpretation künstlerischer Texte - mit lokalen Plausibilitäten argumentiert werden.

Eine Kombination der vorgeschlagenen Kategorien ergibt die folgenden vier Gruppen zur Einteilung von Elementen innerhalb fiktionaler Welten im Hinblick auf deren real-fiktionales Weltvergleichsverhältnis:

\begin{tabular}{lll}
\hline $\begin{array}{l}\text { GRAD DES } \\
\text { VERHÄLTNISSES }\end{array}$ & $\begin{array}{l}\text { Allgemein } \\
\text { (Weltwissen) }\end{array}$ & $\begin{array}{l}\text { Konkret } \\
\text { (Fakten) }\end{array}$ \\
\hline $\begin{array}{l}\text { VERT DES } \\
\text { Übereinstimmung }\end{array}$ & Realistik & Faktik \\
\hline Abweichung & Fantastik & Kontrafaktik \\
\hline
\end{tabular}

$\begin{array}{ll}\text { Realistik: } & \text { Allgemeine Vereinbarkeit von Weltwissen und fiktionaler } \\ & \text { Welt (Analogie zwischen Realität und fiktionaler Welt; } \\ & \text { Mögliches) } \\ \text { Fantastik: } & \begin{array}{l}\text { Allgemeine Nicht-Vereinbarkeit von Weltwissen und } \\ \text { fiktionaler Welt (Abweichung von allgemeinen Annahmen } \\ \end{array} \\ & \text { über die Realität; Unmögliches) }\end{array}$

404 Die Trennung zwischen konkreten und allgemeinen Aspekten der Enzyklopädie entspricht in etwa der von Thomas Zabka vorgeschlagenen Trennung von „Alltagswissen“ und „Sachwissen“. Vgl. Thomas Zabka: Pragmatik der Literaturinterpretation. Theoretische Grundlagen - kritische Analysen. Tübingen 2005, S. 34-36. Allerdings weist auch Zabka darauf hin, dass die Grenzen zwischen Alltagswissen und Sachwissen fließend sind. Vgl. ebd., S. 35, Anm. 17.

405 In ähnlicher Weise konstatiert etwa Brian McHale zu den Fiktionalisierungslizenzen des ,traditionellen“ historischen Romans: „Slippery though they may be, we do operate with intuitions about what is accepted historical 'fact' and how far any fictional version deviates from that 'fact'.“ (McHale: Postmodernist Fiction, S. 87). 
Kontrafaktik:

Faktik:
Konkrete Nicht-Vereinbarkeit von Weltwissen und fiktionaler Welt (Abweichung von realweltlichen Einzelinformationen; bestimmte Negation ${ }^{406}$ )

Konkrete Vereinbarkeit von Weltwissen und fiktionaler Welt (Übereinstimmung von Realität und fiktionaler Welt)

Realistik zeichnet sich demzufolge dadurch aus, dass die fiktionale Welt mit der realen Welt in einem Verhältnis der Analogie steht: Was innerhalb der Diegese geschieht, könnte prinzipiell auch in der realen Welt geschehen, da dieses Geschehen keinen grundlegenden Annahmen über das Wesen der Realität zuwiderläuft. Die Beziehungen des realistischen Erzählens zur tatsächlichen Realität sind dabei eher allgemeiner Natur, insofern sie sich auf wenig spezifische Annahmen über die Realität beziehen. Gegenüber der Frage, ob die Gegenstände des realistischen Erzählens ein konkretes Pendant in der Realität haben, herrscht im typischen Rezeptionsmodus fiktional-realistischer Texte Gleichgültigkeit: Anna Karenina etwa ist eine realistische Figur, unabhängig davon, ob es eine einzelne Frau mit den Eigenschaften der von Tolstoi beschriebenen Figur gegeben hat oder nicht. ${ }^{407}$

Auch für die Fantastik ist eine allgemeine Vereinbarkeit von fiktionaler Welt und Realität Definitionskriterium, nur dass diese Vereinbarkeit hier eben nicht gegeben ist: Fantastische Elemente sind mit Wahrheitsannahmen über das Wesen der realen Welt grundsätzlich inkompatibel. ${ }^{408}$ Um eine Formulierung Renate Lachmanns aufzunehmen: Fantastische Texte „überschreiten die

406 Eine Verbindung zwischen Hegels Konzept der bestimmten Negation und dem kontrafaktischen Denken schlägt auch Slavoj Žižek vor: Disparities, S. 292.

407 Hierzu bemerkt Hoops: „Der Wirklichkeitsgehalt eines fiktionalen Textes bzw. bestimmter Teile eines solchen ist im Falle ,wahrscheinlicher` Geschichten für den Leser in vielen Fällen belanglos, da für ihn die Fiktivität oder Nichtfiktivität bestimmter Individua oder Generalia keinerlei Informationswert besitzt.“ (Hoops: Fiktionalität als pragmatische Kategorie, S. 311).

408 Die Trennung von Fantastischem und Wunderbarem, die in einigen Fantastik-Theorien eine Rolle spielt, kann an dieser Stelle unberücksichtigt bleiben. Auch das Fantastische, im Sinne Todorovs als Unsicherheit hinsichtlich der natürlichen Erklärbarkeit eines bestimmten Sachverhalts verstanden, lässt sich schließlich nur dadurch bestimmen, dass man zumindest zeitweise die Deutung des Geschehens als tatsächlich übernatürlich respektive wunderbar zulässt. Etwas zugespitzt ließe sich formulieren: Das Wunderbare kommt mitunter ohne das Fantastische aus - so etwa im Märchen oder in der Fantasy -, die Fantastik hingegen kann nie ganz auf das Wunderbare verzichten. Vgl. Wünsch: Die Fantastische Literatur der Frühen Moderne (1890-1930), S. 8. Da der Begriff der Fantastik implikationsärmer erscheint, sich besser an die englischsprachige Forschung anschließen lässt und in der Fiktionstheorie auch bereits besser eingeführt ist als der Begriff des Wunderbaren, soll im Folgenden ausschließlich von Fantastik die Rede sein. 
Erfordernisse der mimetischen Grammatik“. ${ }^{409}$ Dabei hypostasieren fantastische Texte nicht den Fall, dass beispielsweise dieser oder jener konkreten, realweltlichen Person innerhalb der fiktionalen Welt magische Kräfte zu Gebote stünden; vielmehr stellen fantastische Texte die Prämisse auf, dass innerhalb der fiktionalen Welt ganz generell Personen vorkommen können, die über derartige Kräfte verfügen. Fantastische Welten beziehen sich qua Abweichung also nicht auf einzelne Fakten der realen Welt, sondern auf deren allgemeine Realitätsstruktur. Entsprechend kann man hier, ähnlich wie bei der Realistik, auch nicht sinnvollerweise von konkreten Realitätsreferenzen sprechen. ${ }^{410}$

Erst im Falle der Kontrafaktik werden konkrete realweltliche Informationen, eben die Fakten, bedeutsam: Kontrafaktische Elemente beziehen sich, anders als realistische oder fantastische Elemente, nicht auf generelle Annahmen über das Wesen der Realität, sondern auf spezifisches Faktenmaterial, etwa auf historische Fakten (in der Alternativgeschichte), auf Eigenschaften bestimmter Personen (beispielsweise in der Satire oder im Schlüsselroman) oder auf gewisse politische oder gesellschaftliche Entwicklungen (in der Dystopie und Utopie). ${ }^{411}$ Wie bei der Fantastik werden auch im Falle der Kontrafaktik Realitätsannahmen allerdings nicht narrativ reproduziert, sondern es wird gerade von bestimmten Realitätsannahmen abgewichen. Da im Falle der Kontrafaktik auf konkrete Einzelelemente der Enzyklopädie und nicht nur auf allgemeine Realitätsannahmen Bezug genommen wird, kann hier - anders als bei Elementen der Realistik oder Fantastik erstmals von Realitätsreferenzen im engeren Sinne gesprochen werden. ${ }^{412}$

Die vierte mögliche Konfiguration betrifft Elemente in fiktionalen Texten, die in konkreter Weise auf realweltliche Fakten referieren. Man könnte hier von

409 Renate Lachmann: Erzählte Phantastik. Zur Phantasiegeschichte und Semantik phantastischer Texte. Frankfurt a. M. 2002, S. 10.

410 Die Aussage, dass in der Realistik und Fantastik konkrete Realitätsreferenzen keine Rolle spielen, steht dabei in keinem Widerspruch zu der Annahme, dass sinnvolle sprachliche Äußerungen immer referieren. Selbstverständlich weisen auch Texte der Realistik oder Fantastik eine Referenzfunktion auf - nur referieren sie eben nicht auf konkrete Einzelinformationen der realen Welt. 411 Diese (fehlende) Spezifität der Realitätsreferenz lässt sich bereits an den Begriffen Realistik und Kontrafaktik ablesen: Während das ,Reale‘ ein eher allgemeines Realitätsverständnis andeutet, erfordert ein ,Fakt‘ immer einen gewissen Grad an Spezifität, um überhaupt als solcher gelten zu können. In ähnlicher Weise konstatieren Johannes Rhein, Julia Schumacher und Lea Wohl von Haselberg in Bezug auf die Faktenbasis kontrafaktischer Geschichtsdarstellungen: „Mit dem Faktischen sind nur in Ausnahmefällen überhistorische und allgemeine Prinzipien wie etwa Naturgesetze gemeint; in der Regel liegt ein verzeitlichtes Konzept von Tatsächlichkeit zugrunde“ (Rhein / Schumacher / Wohl von Haselberg: Einleitung, S. 25).

412 In vergleichbarer Weise bemerkt Zabka: „Man kann vermuten, dass der Bezug auf Sachwissen häufig stark referentiell, der Bezug auf Alltagswissen und Sinnwissen häufig schwach referentiell ist.“ (Zabka: Pragmatik der Literaturinterpretation, S. 37). 
,faktischen“ Elementen oder, um diesen Begriff einzuführen, von Elementen der Faktik sprechen. Typische Beispiele wären Referenzen auf konkrete Orte, historische Daten oder historische Persönlichkeiten, die unverändert in die literarische Fiktion übernommen werden. ${ }^{413}$ In fiktionalen Texten, die komplett aus faktischen Elementen bestehen, bei denen also an jeder Stelle erkannt werden soll, dass die fiktionale Welt mit der realen Welt übereinstimmt, läge der Anteil fiktiver Elemente bei null. Allerdings stellen solche Texte - falls sie überhaupt existieren auffällige Ausnahmen dar. Bei fiktionalen Texten, die in ihrer Gesamtheit auf konkreten Realitätsreferenzen beruhen und bei denen diese Realitätsreferenzen auch als solche erkannt werden sollen, würde sich die Frage aufdrängen, worin hier überhaupt noch den qualitativen Mehrwert fiktionalen Erzählens bestünde (genau diese Frage bildet eines der Grundprobleme aller literarischen Dokumentarismen). Legt man ein pragmatisches Fiktionalitätsverständnis zugrunde, so lässt sich Fiktionalität zwar nicht direkt auf Fiktivität zurückführen; das bedeutet jedoch nicht, dass nicht doch eine gewisse Korrelation zwischen den beiden Bereichen bestünde: Fiktionale Texte, die gänzlich ohne fiktive Elemente auskommen, sind überaus selten (wofern man solchen Texten überhaupt noch die Eigenschaft der Fiktionalität zugestehen will). ${ }^{414} \mathrm{Zu}$ denken wäre etwa an manche dokumentarische Texte, beispielsweise an Truman Capotes Tatsachenroman In Cold Blood, oder an Peter Handkes literarisches poème trouvé Die Aufstellung des 1. FC Nürnberg am 27.1.1968 (das man aber wohl nur noch eingeschränkt als fiktional bezeichnen kann). ${ }^{415}$

413 Faktische Elemente entsprechen damit in etwa dem, was Parsons als immigrant objects bezeichnet. Vgl. Parsons: Nonexistent Objects, S. 51.

414 Zipfel kommt zu dem Schluss: „Fiktivität und Fiktionalität können im Hinblick auf ihre Bedeutung und ihr jeweiliges Bezugsfeld klar unterschieden werden; sie sind jedoch eng miteinander verknüpft und bedingen sich in gewisser Weise gegenseitig.“ (Zipfel: Fiktion, Fiktivität, Fiktionalität, S. 165) Ob Fiktivität als notwendiges Bestimmungskriterium für Fiktionalität gelten kann, bildet einen Streitpunkt der fiktionstheoretischen Diskussion. Vgl. Zipfel: Imagination, fiktive Welten und fiktionale Wahrheit, S. 51.

415 Man könnte einwenden, dass ein vollständig ,realitätsdeckendes‘ Erzählen schon allein aufgrund der Notwendigkeit einer bestimmten medialen - und das heißt auch immer: selektiven Darstellung der Realität gar nicht möglich ist, sodass es ,faktische‘ Texte ohnehin nicht geben kann. Fehlende Vollständigkeit oder eine formale Bearbeitung von Faktenmaterial führen allerdings noch nicht zwingend zu Fiktivität; es kann nicht ausgeschlossen werden, dass jene Aspekte, die in einem bestimmen künstlerisch-fiktionalen Medium überhaupt dargestellt werden können, tatsächlich vollständig auf Fakten der realen Welt referieren. Auch faktuale narrative Darstellungen sind schließlich niemals formal neutral oder vollständig in dem Sinne, dass sie das von ihnen Dargestellte in sämtlichen Details absolut realitätsdeckend wiedergeben würden. Dennoch würde man beispielsweise eine Wegbeschreibung oder einen Zeitungsbericht - beides zweifellos hochgradig selektive Darstellungen der Realität - ihrem Inhalt nach kaum als fiktiv klassifizieren. 
Hinsichtlich der unterschiedlichen Konkretheit von Realitätsbeziehungen in den vier beschriebenen Modi des real-fiktionalen Weltvergleichs lässt sich festhalten: In der Realistik und in der Fantastik wird qua Übereinstimmung respektive Abweichung auf allgemeine Wahrheiten Bezug genommen, in der Faktik und Kontrafaktik hingegen wird qua Übereinstimmung respektive Abweichung auf konkrete Fakten referiert. ${ }^{416}$

$\mathrm{Zu}$ beachten ist, dass bei den beschriebenen real-fiktionalen Weltvergleichsverhältnissen grundsätzlich von bereits interpretierten Texten respektive deutungsrelevanten Elementen innerhalb fiktionaler Texte ausgegangen wird. Insignifikante Fehler in fiktionalen Texten bleiben also erneut unberücksichtigt. Verlegt man solchermaßen die Entscheidung darüber, ob eine realistische, fantastische, kontrafaktische oder faktische Textpassage vorliegt, auf die Ebene der Interpretation, so wird man letztlich niemals beweisen können, dass ein bestimmter Modus des real-fiktionalen Weltvergleichs vorliegt - in vergleichbarer Weise, wie man das Vorliegen eines Sonetts durch Beschreibung seiner äußeren Form und seines Reimschemas beweisen könnte -, einfach deshalb, weil Vorliegen oder Abwesenheit eines bestimmten Weltvergleichsverhältnisses nicht anhand manifester Texteigenschaften nachgewiesen werden kann. Bei den vier vorgeschlagenen real-fiktionalen Weltvergleichsverhältnissen handelt es sich nicht um objektive Texteigenschaften, sondern um Deutungshypothesen, die jeweils der hermeneutisch-interpretatorischen Plausibilisierung bedürfen. ${ }^{417}$ Die Beantwortung der Frage, welches der vier Weltvergleichsverhältnisse vorliegt, hängt also

416 Die zentralen Oppositionsbildungen der vorgeschlagenen Einteilung fiktionaler Texte ließen sich zwecks Verdeutlichung auch allein mittels binärer terminologischer Oppositionen anzeigen. Die Gegenüberstellung Realistik/Fantastik könnte versuchsweise als Realistik/KontraRealistik reformuliert werden. Demgegenüber können solche fiktionalen Texte, die sich auf konkrete Fakten beziehen, in Texte der Kontrafaktik und Texte der Faktik unterteilt werden. Allerdings soll diese ungewohnte, forciert-systematische Terminologie im Verlauf der Arbeit nicht weiter mitgeführt werden.

417 Paul Ricœur bezeichnet das Zusammenspiel von Interpretationshypothese und ihrer Validierung als „Mikrodialektik, die bei der Lösung der lokalen Rätsel eines Textes am Werk ist. [...] [D]ie Verfahren der Validierung [sind] eher mit der Logik der Wahrscheinlichkeit als mit einer Logik der empirischen Verifikation verwandt.“ (Paul Ricœur: Die Metapher und das Hauptproblem der Hermeneutik. In: Ders.: Vom Text zur Person. Hermeneutische Aufsätze (1970-1999). Hamburg 2005, S. 109-134, hier S. 118) Auch Gabriel betont: „Geltung in der Literaturwissenschaft kann [...] weder danach bemessen werden, ob Falsifikationsversuche gescheitert sind, noch danach, ob ein deduktiver Beweis vorliegt. Wir haben es hier mit Indizienbeweisen zu tun, die mehr oder weniger plausibel sind. Es geht, insbesondere bei der Interpretation von Texten, darum, Evidenzen für ein bestimmtes Verständnis beizubringen." (Gabriel: Wie klar und deutlich soll eine literaturwissenschaftliche Terminologie sein?, S. 123). 
letztlich von der interpretatorischen Optik ab: Je nachdem, in welcher Richtung die interpretatorischen Präferenzen liegen und wie im Rahmen einer konkreten Textdeutung argumentiert wird, kann die Zuordnung einzelner Erzählelemente zur Realistik, Fantastik, Kontrafaktik oder Faktik mitunter sehr unterschiedlich ausfallen. Das bedeutet freilich nicht, dass diese Zuordnungen willkürlich wären: Unter Voraussetzung eines bestimmten Realitätsverständnisses, einer bestimmten Enzyklopädie und eines bestimmten Interpretationsansatzes kann die Zuordnung einzelner Elemente zu einem der vier vorgestellten Weltvergleichsverhältnisse durchaus quasi-objektiv ausfallen. So dürfte etwa ein Drache in einem Roman der Gegenwart fast immer als fantastisches Element klassifiziert werden.

Da es sich bei der vorgeschlagenen Systematik um eine Ordnung der realfiktionalen Weltvergleichsverhältnisse in Bezug auf konkrete Elemente handelt, muss nicht davon ausgegangen werden, dass Texte in ihrer Gesamtheit einem einzigen der vier Typen zuzuschlagen sind. Im Gegensatz zu Begriffen wie Fantasy oder Alternate History bezeichnen Realistik, Fantastik, Kontrafaktik und Faktik keine Genres, sondern vier basale Möglichkeiten des real-fiktionalen Weltvergleichs. In umfänglicheren fiktionalen Werken wird in aller Regel eine Mischung verschiedener Weltvergleichsverhältnisse vorliegen. So dürfte es kaum längere fiktionale Erzähltexte geben, die sich nicht zumindest streckenweise des Weltvergleichsverhältnisses der Realistik bedienen.

Tatsächlich besteht in den allermeisten fiktionalen Texten ein deutlicher Überhang realistischer Elemente: ${ }^{418}$ Selbst in Texten des Fantasy-Genres werden nur selten Annahmen über basale psychische Prozesse der - letztlich fast immer anthropomorph konzipierten ${ }^{419}$ - Handlungsträger variiert; auch werden selten grundlegende Naturgesetze wie die Schwerkraft oder der monodirektionale Verlauf der Zeit ausgesetzt. Selbst einzelne fantastische Elemente, wie sie für die Zuordnung eines Textes zum Genrebereich der Fantasy ausschlaggebend sein können, bewegen sich also meist vor einem weitgehend realistischen Hintergrund. Fantastische Texte, in denen dies nicht der Fall ist, die also gewissermaßen in toto fantastisch sind - wie etwa Paul Scheerbarts radikaler Science-Fiction-Roman Lesabéndio (1913) - bilden eine entschiedene Ausnahme.

In dominant realistischen Texten wiederum wird sehr häufig - zumindest streckenweise - auf das Weltvergleichsverhältnis der Faktik zurückgegriffen,

418 Zum „quantitativen Übergewicht [...] nichtfiktionaler Konzepte in fiktionalen Texten“ siehe Blume: Fiktion und Weltwissen, S. $83 \mathrm{f}$.

419 Für die meisten Figuren in narrativen Medien gilt: „The participants involved in the actions and events related are animate, usually humans [...]. If participants are not humans, as, for example, in fables or fairy tales, they still possess human qualities and act like humans.“ (Gülich / Quasthoff: Narrative Analysis, S. 171). 
etwa wenn im historischen Roman auf reale politische Ereignisse, konkrete Orte oder auf realhistorische Personen Bezug genommen wird. ${ }^{420}$ Legt man nicht das beschriebene, spezifisch fiktionstheoretische Verständnis von ,Realistik‘, sondern ein gängigeres Verständnis von ,Realismus‘ an - im Sinne dominant realistischer Erzählverfahren, durch die sich eine bestimmte Gruppe von Texten auszeichnet -, so wird man feststellen, dass eine große Anzahl von Texten, die für gewöhnlich dem ,Realismus‘ zugeschlagen werden, sich hinsichtlich ihrer real-fiktionalen Weltvergleichsverhältnisse gerade einer Mischung aus Realistik und Faktik bedienen.

Hinsichtlich der Frage der Referenz ergibt sich hieraus für eine Differenzierung von Texten des Realismus (nicht der Realistik!) und Texten der Kontrafaktik folgende Konsequenz: Texte des Realismus können in konkreter Weise auf die reale Welt referieren; dies wäre gerade der Fall bei faktischen Elementen innerhalb von Texten, die ansonsten im Modus der Realistik erzählt sind (typische Beispiele umfassen etwa die Nennung von Städtenamen oder von bekannten historischen Ereignissen in realistischen Texten). Texte der Kontrafaktik hingegen müssen in konkreter Weise auf die reale Welt Bezug nehmen. ,Realistisches‘ Erzählen kann also schlicht realitätsanalog verfahren und gegebenenfalls nur punktuell auf konkrete (faktische) Faktenelemente verweisen; die Kontrafaktik hingegen bedarf notwendigerweise - und ungeachtet dessen, dass es sich hier um ein amimetisches Erzählverfahren handelt - eines starken Ankers in der Realität, nämlich der konkreten Fakten, von denen dann in Form von Realitätsvariationen abgewichen wird. Es kann hier noch einmal auf die Relevanz des konkreten Weltvergleichs für die Kontrafaktik - und auch für die Faktik - hingewiesen werden: Während im Fall der Realistik und der Fantastik der wechselseitig-komparative

420 Faktik im strengen Sinne läge allerdings nur dann vor, wenn der - um beim genannten Beispiel zu bleiben - historische Hintergrund oder das historische Personal eines historischen Romans von der fiktionalen Erzählung vollkommen unberührt blieben. Wenn hingegen etwa in Joseph Roths Radetzkymarsch der fiktive Leutnant Joseph Trotta dem jungen Kaiser Franz Joseph in der Schlacht von Solferino das Leben rettet, liegt hier strenggenommen keine Faktik mehr vor: Zwar handelt es sich sowohl bei Kaiser Franz Joseph als auch bei der Schlacht von Solferino um realhistorische Entitäten; durch den fiktiven Eingriff Joseph Trottas werden diese Entitäten innerdiegetisch allerdings leicht verändert, sodass sie nicht mehr vollständig mit ihren realen Vorbildern übereinstimmen. Das bedeutet freilich nicht, dass realweltliches Wissen für die Deutung von Figuren wie denen in Roths Roman irrelevant wäre. Auch kann man die These vertreten, dass die Faktenabweichung in einem Fall wie dem genannten derart gering ist, dass sie interpretatorisch nicht berücksichtigt werden muss. Insofern könnte man bei Kaiser Franz Joseph in Roths Radetzkymarsch weiterhin von einem (faktischen) immigrant object und nicht von einem (kontrafaktischen) surrogate object sprechen. Vgl. Zipfel: Fiktion, Fiktivität, Fiktionalität, S. 100. 
Weltbezug eher vage ist, also kein klares Faktum der realen Welt als Bezugspunkt identifiziert werden kann, gibt es ein solches Faktum im Fall der Faktik durchaus; nur wird dieses Faktum innerdiegetisch eben nicht variiert. Eine konkrete variierende Faktenreferenz liegt einzig im Fall der Kontrafaktik vor.

Wie steht es nun aber um Erzählelemente, die sich dem ersten Anschein nach zweien der genannten Modi gleichzeitig zuordnen lassen? Kann dasselbe Element gleichzeitig kontrafaktisch und fantastisch, realistisch und faktisch sein?

Zur Beantwortung dieser Frage sei ein konkretes Beispiel diskutiert: Viele der frühen Interpreten von J. R. R. Tolkiens The Lord of the Rings, eines Romans, der teilweise während des Zweiten Weltkriegs entstand, meinten in der Geschichte des Ringkriegs eine Allegorie auf den realen Krieg zu erkennen. Speziell der titelgebende Herr der Ringe, der dunklen Herrscher Sauron - ein körperloses, fantastisches Wesen in Tolkiens fiktionaler Welt -, wurde als Chiffre für Adolf Hitler interpretiert. Tolkien selbst distanzierte sich im Vorwort zur revidierten Ausgabe von 1966 entschieden von derartigen allegorischen Deutungen seines Werks:

As for any inner meaning or 'message', it has in the intention of the author none. It is neither allegorical nor topical. [...] Its sources are things long before in mind, or in some cases already written, and little or nothing in it was modified by the war that began in 1939 or its sequels. [...] I cordially dislike allegory in all its manifestations, and always have done so since I grew old and wary enough to detect its presence. I much prefer history, true or feigned, with its varied applicability to the thought and experience of the readers. I think that many confuse 'applicability' with 'allegory'; but the one resides in the freedom of the reader, and the other in the purposed domination of the author. ${ }^{421}$

Die Unterscheidung zwischen dem, was Tolkien die ,Freiheit des Lesers' versus die ,zweckgerichtete Entscheidung des Autors' nennt, ließe sich im gegebenen Fall auf eine von Umberto Eco vorgeschlagene Unterscheidung beziehen, nämlich auf diejenige zwischen der ,Verwendung ' und der ,Interpretation“ eines Textes: Ein beliebiger Umgang mit einem (literarischen) Text könne zwar nicht verboten werden (Eco führt als Beispiel an, dass man aus den Seiten eines Buches auch einen Joint drehen kann); um eine eigentliche Interpretation dieses Textes wird es sich jedoch nur dann handeln, wenn gewissen Anforderungen der hermeneutischen Billigkeit entsprochen wird. ${ }^{422}$ Im Falle des Lord of the Rings scheint zumindest der Autor der Meinung gewesen zu sein, dass der Text keinen hinreichenden Anlass für eine allegorische Lesart bietet, dass also eine allegorische (oder auch kontrafaktische) Rückbindung des Romans an den realen

421 J. R. R. Tolkien: The Lord of the Rings. New York 1987, S. 4 f.

$422 \mathrm{Vgl}$. Eco: Lector in fabula, S. 72-74. 
Krieg zu keiner legitimen Interpretation desselben führen würde. Tolkien zufolge handelt es sich bei den mythischen Figuren seines Romans also eindeutig um fantastische Elemente im Sinne der obigen Definition.

Nun muss den Interpretationen literarischer Texte durch ihre eigenen $\mathrm{Au}$ toren freilich nicht gefolgt werden. Angenommen, es kämen berechtigte Zweifel an Tolkiens Lesart des Lord of the Rings auf und man würde zu dem Schluss gelangen, dass der dunkle Herrscher Sauron durchaus eine allegorische Darstellung Adolf Hitlers bildet: Würde das fantastische Element dadurch zu einem kontrafaktischen Element? Und wenn ja, ginge hierdurch der fantastische Charakter dieses Elements verloren?

Kontrafaktische Elemente können, wie im Rahmen der Diskussion des Kompositionalismus ausgeführt wurde, als real-fiktive Hybridobjekte verstanden werden: Einerseits modifizieren solche Elemente realweltliche Fakten, andererseits behalten sie zugleich hinreichend viele Teilaspekte derselben Fakten bei, um die konkrete Faktenreferenz weiterhin erkennen zu lassen. Wollte man nun Sauron mit Hitler parallelisieren, so scheinen zunächst beide Bedingungen erfüllt zu sein: Selbstverständlich sind die Objekte nicht - im Sinne der Faktik - identisch: In den allermeisten ihrer Eigenschaften (Name, Aussehen, magische Fähigkeiten etc.) weichen sie deutlich voneinander ab. Allerdings lassen sich durchaus Übereinstimmungen zwischen beiden ausmachen, namentlich ein totalitäres Machtstreben, rücksichtslose Grausamkeit sowie die Stellung als (militärischer) Führer eines kriegerischen, imperial ambitionierten Reiches. Wollte man diese Aspekte als Fakten akzeptieren, so könnte man tatsächlich von einer kontrafaktischen Variation Hitlers im Lord of the Rings sprechen, wobei das kontrafaktisch variierte Element innerhalb der fiktionalen Welt zugleich ein fantastisches Element wäre.

Im beschriebenen Fall sind die relevanten Übereinstimmungen jedoch eher allgemeiner Natur; sie erfüllen also nur sehr eingeschränkt die Forderung nach Spezifität, welche an die Fakten der Kontrafaktik stets zu stellen ist, damit ein konkreter Faktenbezug auch zuverlässig identifiziert werden kann. Angesichts der erwähnten Eigenschaften könnte Tolkiens Sauron schließlich auch als kontrafaktische Variante Cäsars gedeutet werden. Eine kontrafaktische Interpretation erscheint im beschriebenen Fall also keineswegs zwingend.

Unabhängig davon, wie man im konkreten Fall urteilen wird, lässt sich bezüglich der Frage einer Zuordnung desselben Elements zur Fantastik oder zur Kontrafaktik festhalten, dass ein fantastisches Element dadurch, dass man es als kontrafaktisches Element interpretiert, keineswegs seine Eigenschaft als fantastisches Element verliert - so wie ein faktisches Element ja auch immer ein realistisches Element sein kann und in gewissem Sinne sein muss. (Faktik impliziert Realistik und schließt Fantastik aus, weil nichts konkret wahr sein kann, was gegen allgemeine Realitätsannahmen verstößt. Kontrafaktik hingegen impliziert 
kein anderes Weltvergleichsverhältnis, kann sich also sowohl mit Fantastik als auch mit Realistik überlagern. Allerdings schließt Kontrafaktik Faktik aus, weil eine Aussage nicht zugleich realweltlich wahr und realweltlich falsch sein kann.) Tatsächlich wird die Faktenabweichung, die dem kontrafaktischen Erzählen zugrunde liegt, in einem Fall wie dem beschriebenen von der allgemeineren fantastischen Faktenabweichung gleichsam partiell mitübernommen. Anders ausgedrückt: Kontrafaktische Texte können sich durchaus bei den Formen der allgemeineren Realitätsabweichung der Fantastik ,bedienen', um konkret-kontrafaktische Faktenvariationen ins Werk zu setzen (was freilich nicht bedeutet, dass kontrafaktische Elemente zugleich immer auch fantastisch wären; Faktenvariationen können auch innerhalb des Spielraums realistischen Erzählens vorgenommen werden). Würden also etwa in einer fiktionalen Erzählung einer bekannten Person des öffentlichen Lebens - etwa einer Politikerin - Flügel wachsen, so handelte es sich bei dieser Figur sowohl um ein fantastisches Element (Menschen haben allgemein in der realen Welt keine Flügel) als auch um ein kontrafaktisches Element (dieser konkrete Mensch hat in der realen Welt keine Flügel). Ein alleiniger Blick auf die Ontologie der Erzählgegenstände erlaubt in derartigen Fällen keine Unterscheidung zwischen den beiden möglichen Weltvergleichsverhältnissen; die Frage, ob man es eher mit einem fantastischen oder eher mit einem fantastisch-kontrafaktischen Element zu tun hat, entscheidet sich hier vor allem auf der Ebene der Interpretation. Liest man also etwa Sauron als fantastisches Element, so wird man im Rahmen einer Interpretation des Lord of the Rings weitgehend von Rückgriffen auf die Realhistorie zur Stützung oder Entwicklung der eigenen Interpretationshypothesen absehen müssen; liest man Sauron hingegen als ein fantastisch-kontrafaktisches Element, bei dem die Fantastik lediglich als eine von diversen Möglichkeit der Faktenvariation genutzt wird, so tritt die Geschichte des Zweiten Weltkriegs als relevanter Interpretationskontext hinzu, was natürlich erhebliche Konsequenzen für die Interpretation des Textes nach sich zieht.

Das diskutierte Beispiel zeigt, dass die Entscheidung für eines der vier Weltvergleichsverhältnisse weit mehr als bloße terminologische Makulatur ist: Tatsächlich erweist sich die Entscheidung über das jeweils vorliegende oder plausibilisierbare real-fiktionale Weltvergleichsverhältnis als in hohem Maße relevant für die Interpretation des jeweiligen literarischen Textes. ${ }^{423}$ Indem sich faktische und kontrafaktische Elemente auf konkrete Fakten der realen Welt beziehen, ermöglichen sie

423 Hoops bemerkt in ähnlicher Weise: „Je nachdem, welche Intention der Leser hypothetisch ansetzt, erhalten Wirklichkeitsabweichungen bzw. die (Nicht-)Fiktivität bestimmter Elemente eine unterschiedliche Relevanz, und umgekehrt ist die Leserhypothese abhängig von der Relevanz, die der Leser der Fiktivität bzw. Nichtfiktivität bestimmter Elemente zuschreibt“ (Hoops: Fiktionalität als pragmatische Kategorie, S. 310). 
eine spezifische Form der Kontextselektion, nämlich eine interpretatorische Anbindung an konkrete Fakten der realen Welt, wie sie bei realistischen und fantastischen Elemente nicht gegeben ist. Durch ihre Referenz auf realweltliche Fakten etablieren faktische und kontrafaktische Elemente also eine enge Kopplung von realer und fiktionaler Welt, sodass es besonders naheliegend erscheint, bei der Deutung der jeweiligen fiktionalen Werke die vorgängige Bedeutung der referentialisierten Fakten in ihrem ursprünglichen, realweltlichen Kontext mit zu berücksichtigen. Diese konkrete Text-Kontextverknüpfung kann weitreichende Konsequenzen gerade für die politische Deutung eines literarischen Textes haben, hängen politische Lesarten doch wesentlich davon ab, dass und in welcher Weise literarische Texte auf die reale Welt bezogen werden. Man führe sich beispielsweise unterschiedliche Formen der fiktionalen Kriegsdarstellung vor Augen: Die realistischen oder faktischen Kriegsdarstellungen in Erich Maria Remarques Im Westen nichts Neues oder in Ernst Jüngers In Stahlgewittern (wobei der Fiktionalitätsstatus von Jüngers erster Buchpublikation strittig ist) wird man interpretatorisch sowie politisch-normativ anders bewerten als die kontrafaktische Darstellung des Zweiten Weltkriegs in Quentin Tarantinos Inglourious Basterds und diese wiederum anders als die fantastischen Kriegsdarstellungen in den Star Wars-Filmen, unter anderem deshalb, weil letztere keine eindeutige Referenzen auf reale Kriege etablieren, wie es realistisch-faktische und auch kontrafaktische Kriegsdarstellungen durchaus tun.

Streng ontologisch betrachtet sind kontrafaktische und fantastische Elemente gleichermaßen fiktiv, da beide Klassen von Elementen in der Realität nicht vorkommen (entsprechend wird bei den kontrafaktischen Konditionalen der analytischen Philosophie auch nicht unterschieden zwischen solchen Aussagen, die mit allgemeinen Annahmen über die Realität unvereinbar sind, und solchen, die einzelne Fakten variieren). Im Fall von Literatur und Literaturwissenschaft erscheint es jedoch sinnvoll, zwischen allgemeiner und konkreter Realitätsabweichung, also zwischen Fantastik und Kontrafaktik, zu unterscheiden, da sich hier jeweils sehr unterschiedliche Forderungen für die Interpretationspraxis ergeben: Fantastische Elemente können weitgehend werkimmanent interpretiert werden, da eine bloß allgemeine Faktenabweichung eben auch keine spezifischen Fakten als relevante Interpretationskontexte selegiert. Bei kontrafaktischen Elementen hingegen muss der implizite Faktenbezug der Texte, in denen diese Elemente vorkommen, erkannt und interpretatorisch produktiv gemacht werden.

DiekonkreteFaktenbindung, welche unter anderem die Kontrafaktikauszeichnet, ist nicht zuletzt relevant für eine politisch orientierte Deutung solcher Texte, deren dominantes Weltvergleichsverhältnis die Fantastik bildet. Da Kontrafaktik und Fantastik prinzipiell miteinander kombinierbar sind, bietet sich für fantastische Texte die Möglichkeit, einen konkreten Weltbezug ,hinzuzufügen‘, 
indem ihre fantastischen Elemente zusätzlich mit kontrafaktischen Referenzen unterlegt werden (ein kontrafaktisch-fantastischer Herr der Ringe würde auf Hitler verweisen, ein ausschließlich fantastischer Herr der Ringe hingegen nicht). Es eröffnet sich hier also die Möglichkeit einer Kunst, deren Darstellungsverfahren einerseits dezidiert amimetisch sind, die aber andererseits qua Kontrafaktik einen klaren Bezug zur Realität wahrt. Der Vorwurf des Eskapismus, wie er gegen fantastische Genres wie Fantasy und Science-Fiction immer wieder erhoben wurde, ließe sich entsprechend dadurch abschwächen, dass man für die jeweiligen Werke kontrafaktische Lesarten zu plausibilisieren sucht. ${ }^{424}$

\subsection{Kontrafaktik als genreunabhängige Referenzstruktur}

Wie im Rahmen der Forschungsdiskussion bereits umfassend dargelegt wurde, hat die bisherige literaturwissenschaftliche Forschung das literarische kontrafaktische Erzählen fast ausschließlich im Zusammenhang mit dem historischen Erzählen, insbesondere dem historischen Roman diskutiert. Eine solche thematische Einschränkung erscheint durchaus legitim, wofern das zentrale Untersuchungsinteresse auf Fragen der Genre-Konstitution und Genre-Entwicklung der Alternate History liegt. Geht man hingegen von der basalen Referenzstruktur literarischen kontrafaktischen Erzählens aus, so verpflichtet nichts zu einer starren Kopplung von Kontrafaktik und historischem Erzählen.

Im Folgenden sollen die Vorteile einer referenzstrukturellen, genreunabhängigen Betrachtung der Kontrafaktik erläutert werden. Eine Konzeption der Kontrafaktik als Referenzstruktur ermöglicht vorderhand eine präzise Bestimmung kontrafaktischer Elemente, ehe dann in einem zweiten, abgeleiteten Schritt danach gefragt wird, in welchen Genres Kontrafaktik besonders häufig zum Einsatz kommt und welche medialen Voraussetzungen erfüllt sein müssen, damit sich kontrafaktische, transfiktionale Doppelreferenzen ergeben.

Durch eine Fokussierung der kontrafaktischen Referenzstruktur wird das bisher in der Forschung vorherrschende Verständnis literarischen kontrafaktischen Erzählens gewissermaßen umgekehrt: Gefragt wird nicht mehr danach, in welchen historischen Romanen sich kontrafaktische Elemente finden und ob diese Romane möglicherweise ein eigenes Subgenre des historischen Romans bilden; stattdessen wird zunächst von einer spezifischen Möglichkeit literarischer Texte, auf Welt zu referieren, ausgegangen und erst danach die Frage gestellt, in welchen Genres und

424 Siehe Kapitel 9. Politische Kontrafaktik. 
Einzeltexten diese Referenzstruktur genutzt wird. ${ }^{425}$ Neben die Analyse der spezifischen Referenzstruktur der Kontrafaktik im Genre der Alternate History treten somit Analysen derselben Referenzstruktur etwa in Satiren, Dokufiktionen, Autofiktionen, Utopien und Dystopien oder im Schlüsselroman.

Hinsichtlich der Genretheorie verfolgt die vorliegende Studie insgesamt eine Doppelstrategie: Zunächst werden Genrefragen strategisch zurückgestellt, um ein neutral fiktionstheoretisches Modell der Kontrafaktik $\mathrm{zu}$ entwickeln; in einem zweiten Schritt werden die unterschiedlichen Genres, in denen kontrafaktische Referenzstrukturen gehäuft vorkommen, dann aber durchaus wieder in den Fokus gerückt. Durch die temporäre Suspendierung der Genrefrage kann eine neue, gleichsam vorurteilsfreie Einstellung zur Frage kontrafaktischer Genres gewonnen werden: Eine fiktionstheoretische Betrachtung der Kontrafaktik eröffnet die Möglichkeit, Affinitäten und Strukturanalogien zwischen unterschiedlichen Gruppen von Texten zu erkennen und zu beschreiben, Verbindungen, die bei einer primären Berücksichtigung von Genre-Fragen, wie sie in der bisherigen Kontrafaktik-Forschung dominiert, mitunter verborgen bleiben müssten.

Als einer von wenigen Kontrafaktik-Forschern hat Christoph Rodiek darauf aufmerksam gemacht, dass das kontrafaktische Erzählen kein Genre und keine Textgattung bildet, sondern auf seinem basalen Level durch eine bestimmte „Struktur“ charakterisiert ist:

Die Uchronie ist keine Gattung, vielmehr handelt es sich beim kontrafaktischen Erzählmodus um ein textsortenunabhängiges Darstellungsmuster. Wie bei der Definition der literarischen Phantastik sollte man auch bei der Bestimmung des Uchronischen nicht von vollständigen Texten ausgehen. Denn das Uchronische ist keine Textsorte, sondern eine Struktur bzw. ein Ereigniskontinuum, das als Element in ganz unterschiedliche Textsorten integriert werden kann. ${ }^{426}$

425 Analog spricht Wünsch für das Fantastische auch von einer „Textstruktur“ oder einer „narrative[n] Struktur“ (ebd., S. 8, 16). Der Begriff ,Referenzstruktur“ erscheint für die Kontrafaktik allerdings eher geeignet, da er erstens dem Missverständnis vorbeugt, Kontrafaktik lasse sich allein anhand des Textes - also ohne Ansehung seines epistemischen Kontexts - definieren, und weil er zweitens deutlich macht, dass Kontrafaktik (indirekt) auf konkrete Aspekte der realen Welt, eben die Fakten, referiert, was bei der Fantastik nicht der Fall ist.

426 Rodiek: Erfundene Vergangenheit, S. 27. Auch Holger Korthals distanziert sich bei der Bestimmung der Alternate History von einem Gattungsmodell zugunsten des Fokus auf eine bestimmte „Schreibweise“. Dabei schlägt Korthals vor, „die alternate history über ihr Verhältnis zur Wirklichkeit bzw. zum jeweils herrschenden Konstrukt dessen, was als Wirklichkeit aufgefaßt wird, zu definieren. “ (Holger Korthals: Spekulationen mit historischem Material. Überlegungen zur alternate history. In: Rüdiger Zymner (Hg.): Allgemeine Literaturwissenschaft Grundfragen einer besonderen Disziplin. 2., durchgesehene Aufl. Berlin 2001, S. 157-169, hier S. 159, 161). 
Zwischen den zahlenmäßig dominanten Ansätzen in der bisherigen KontrafaktikForschung einerseits, welche die Kontrafaktik mehr oder weniger eng an den historischen Roman binden, und dem Ansatz der vorliegenden Studie andererseits liegen die Ausführungen Rodieks gewissermaßen in der Mitte: Zwar hält Rodiek, wie bereits durch seine Verwendung des Begriffs des „Uchronischen“ deutlich wird, an den historischen Fakten als Basis der Kontrafaktik fest. Zugleich aber betont er, dass das kontrafaktische Schreiben als „Struktur“ oder „Ereigniskontinuum“ anzusehen sei und entsprechend nicht an eine bestimmte Gattung gebunden werden könne. Sehr zurecht weist Rodiek darüber hinaus auf die Parallele des kontrafaktischen mit dem fantastischen Erzählen hin: Auch das fantastische Erzählen wird häufig im Kontext von Genreüberlegungen beschrieben, kann aber auch - und mitunter plausibler - als Struktur behandelt werden, die in ganz unterschiedlichen Genres und Medien Verwendung findet. ${ }^{427}$

Kontrafaktische Referenzstrukturen kommen, wie im vorigen Kapitel bereits ausgeführt wurde, für gewöhnlich nur bei einzelnen Elementen eines Textes zur Geltung. Bei ,kontrafaktischen Texten' handelt es sich entsprechend nicht um eine streng distinkte Kategorie mit klar abgesteckten Grenzen, sondern eher um einen pragmatischen Vorschlag für die metonymische Gesamtcharakterisierung solcher Texte, in denen kontrafaktische Elemente entweder in hohem Umfang vorliegen oder aber besonders deutungsrelevant sind. ${ }^{428}$ Anders ausgedrückt: Kontrafaktische Texte sind solche, für deren Interpretation eine Berücksichtigung kontrafaktischer Passagen unerlässlich ist oder sich zumindest in hohem Maße anbietet. ${ }^{429}$

427 Vgl. Wünsch: Die Fantastische Literatur der Frühen Moderne (1890-1930), S. 7-17.

428 Siehe Kapitel 4.3.6. Skopus: Kontrafaktische Welten, kontrafaktische Elemente, point of divergence. Eine ähnliche Bestimmungsstrategie verfolgt Darko Suvin in seiner klassischen Studie zur Science-Fiction: „Eine SF-Erzählung ist eine Prosadichtung, in der das SF-Element oder der SF-Aspekt, das Novum, dominierend ist, d. h. so zentral und so wesentlich, daß die ganze Erzähllogik - oder zumindest die vorherrschende Erzählhaltung - von ihm bestimmt wird, ungeachtet aller eventuellen Verunreinigungen.“ (Darko Suvin: Poetik der Science Fiction. Zur Theorie und Geschichte einer literarischen Gattung. Frankfurt a. M. 1979, S. 100 f.).

429 Will man die wissenschaftliche Beschreibungssprache nicht durch permanente Feindifferenzierungen verkomplizieren, so suggeriert sie bedauerlicherweise einen Essentialismus, der mit einem dynamischen und wesentlich rezeptionstheoretischen Modell wie dem vorgestellten strenggenommen nicht vereinbar ist. Die Aussage „Text X ist kontrafaktisch“ müsste im Grunde spezifiziert werden $\mathrm{zu}$ „Text X enthält kontrafaktische Elemente“, was wiederum spezifiziert werden müsste zu „Text X enthält Elemente, die, als kontrafaktische Elemente angesehen, für eine Interpretation des Textes relevant sind“, was wiederum spezifiziert werden müsste zu „Text X enthält Elemente, die von einem Leser mit einem spezifischen epistemischen Horizont als kontrafaktische Elemente identifiziert und in eine schlüssige Gesamtinterpretation des Textes integriert werden könnten “ und so weiter. Um eine konstante Wiederholung derartiger Satzungetüme zu vermeiden, wird im Folgenden weiterhin von „kontrafaktischen Texten“ und „kontrafaktischen Elementen“ 
Der Umstand, dass Gruppenbezeichnungen fiktionaler Texte sich nach Dominanz und Relevanz eines bestimmten real-fiktionalen Weltvergleichsverhältnisses richten - eines Verhältnisses, das gleichwohl nicht das einzige im Text identifizierbare Weltvergleichsverhältnis sein muss -, stellt dabei keineswegs ein Spezifikum kontrafaktischer Texte dar. Auch Texte der Fantastik weisen meist nur einzelne, textuell isolierte fantastische Elemente auf, was jedoch nicht daran hindert, diese Texte in ihrer Gesamtheit als fantastische Texte zu charakterisieren. ${ }^{430}$ Was die Genrefrage betrifft, so verhalten sich fantastische Elemente zum Genre der Fantasy in etwa so wie kontrafaktische Elemente zum Genre der Alternate History (sowie zu einigen anderen Genres): Sie bilden ein notwendiges Definitionskriterium dieser Genres, ohne dass jedoch alle Elemente der jeweiligen Texte der Fantastik respektive Kontrafaktik zugeordnet werden müssten.

Mitunter erscheint es gar nicht sinnvoll, einen Text überhaupt auf ein einziges, dominantes Weltvergleichsverhältnis festzulegen. Ein großer Vorteil einer Strukturbetrachtung der Kontrafaktik besteht darin, dass man nicht zu den typologischen Gesamttext-Rubrizierungen gezwungen ist, wie sie mit Genre-Modellen fast notwendigerweise einhergehen, sondern sich auf die präzise Analyse einzelner Textstellen konzentrieren kann. Ein solches Vorgehen ist hermeneutischinterpretatorisch meist deutlich produktiver und bietet besondere Vorteile bei der Auseinandersetzung mit Werken, die hinsichtlich ihrer Realitätsbezüge eine Mischung unterschiedlicher real-fiktionaler Weltvergleichsverhältnisse aufweisen, etwa Texte, die Genrehybridisierungen vornehmen. So weist der Comic Watchmen von Alan Moore und Dave Gibbons eine ganze Reihe unterschiedlicher Erzählverfahren auf: Erzählt wird - mit Anleihen unter anderem bei der ScienceFiction, der Fantasy und dem historischen Abenteuerroman - die Geschichte zweier Generationen von Superhelden in einem alternativgeschichtlichen Amerika zur Zeit des Kalten Krieges. Eine General-Rubrizierung des Werkes qua Genre oder dominantem Weltvergleichsverhältnis erscheint bei einem solchen Werk wenig sinnvoll. Eine Beschreibung der Kontrafaktik als genreunabhängige Referenzstruktur erlaubt es hier, zunächst die kontrafaktischen Elemente des Werkes $\mathrm{zu}$ identifizieren und sie dann im Anschluss interpretatorisch gegen

die Rede sein, wobei eben immer mitzudenken ist, dass es sich hier nicht um positive Eigenschaften an der Textoberfläche, sondern um interpretatorische Konstrukte handelt.

430 So bemerkt Klaudia Seibel: „[T]hough it is legitimate to characterise texts as a whole as fantastic, even if there is only a single reference to an impossible object or event [...] on a micro level there might be sentences, paragraphs, even whole chapters that by itself have nothing fantastic about them." (Klaudia Seibel: "Read, friend, and enter!” Generic world construction in fantastic texts. In: Pascal Klenke u. a. (Hg.): Writing Worlds. Welten- und Raummodelle der Fantastik. Heidelberg 2014, S. 227-240, hier S. 230). 
anders gelagerte Erzählelemente abzuwägen - und somit der realen Komplexität des Untersuchungsgegenstandes besser Rechnung zu tragen. ${ }^{431}$

\subsection{Kontrafaktische Genres}

Ein Verständnis von Kontrafaktik als Referenzstruktur schließt, wie bereits erwähnt, eine Diskussion kontrafaktischer Genres keineswegs aus. Im Folgenden soll danach gefragt werden, in welchen Genres die transfiktionalen Doppelreferenzen der Kontrafaktik mit besonderer Häufigkeit zum Einsatz kommen und was der Grund für diese Häufung ist. Für die Genres der Alternativgeschichte, des kreativen Dokumentarismus sowie der Dystopie und Utopie wird diese Verbindung von kontrafaktischer Referenzstruktur und Genretheorie im Interpretationsteil der Arbeit noch sehr viel umfänglicher zu erläutern sein. Der besseren Übersichtlichkeit halber soll allerdings bereits an dieser Stelle knapp auf die genannten Genres eingegangen werden.

Die folgende kommentierte Genreliste erhebt keinen Anspruch auf Vollständigkeit. Sie umfasst lediglich eine Reihe von Fällen, bei denen die Frage nach einer möglichen kontrafaktischen Referenzstruktur besonders naheliegend erscheint. Kontrafaktische oder potenziell kontrafaktische Genres sind unter anderem die folgenden:

Alternativgeschichte/Alternate History: Das Genre der Alternativgeschichte oder Alternate History bildet zweifellos das klassische Einsatzgebiet für kontrafaktische Referenzstrukturen in Literatur, Film und Comic. Wie bereits im Rahmen der Forschungsdiskussion ausgeführt wurde ${ }^{432}$, eignen sich historische Fakten in besonderem Maße zur kontrafaktischen Variation, da sie über ein hohes Maß an intersubjektiver Verbindlichkeit verfügen und sich gut narrativieren lassen. Sofern die Lebensläufe bekannter Persönlichkeiten variiert werden, bieten historische Stoffe darüber hinaus den Vorteil, dass die handelnden Individuen meist schon seit längerer Zeit tot sind und insofern von Autorenseite

431 Die Entscheidung darüber, ob überhaupt kontrafaktische Elemente in einem fiktionalen Werk vorhanden sind, beruht selbst bereits auf einer Interpretationsleistung; dies gilt es grundsätzlich zu berücksichtigen, wenn in dieser Arbeit von ,kontrafaktischen Elementen ' die Rede ist. Bei derart offenkundig realitätsvariierenden Sachverhalten, wie sie im Comic Watchmen zu finden sind - namentlich dem Sieg der Amerikaner im Vietnam-Krieg aufgrund des Eingreifens eines durch einen Laborunfall in ein übernatürliches Wesen verwandelten Atomphysikers -, dürfte die Einsicht, dass hier ein Fall von Kontrafaktik vorliegt, allerdings kaum von der Hand zu weisen sein. Vgl. Alan Moore / Dave Gibbons: Watchmen. New York 2014, S. $110-130$.

432 Siehe Kapitel 3.2.3. Kontrafaktisches Erzählen als Genrevariante historischen Erzählens. 
kaum Rücksicht auf die Verletzung von Persönlichkeitsrechten genommen werden muss. ${ }^{433}$

Utopie / Dystopie: Dystopie und Utopie werden im Rahmen dieser Arbeit gemeinsam verhandelt, da ihr Unterscheidungskriterium, die Frage nämlich, ob in den jeweiligen Texten eine positive oder eine negative Gesellschaftsorganisation dargestellt wird, fiktionstheoretisch unerheblich ist (manchmal wird schlicht von Utopie und Anti-Utopie gesprochen); oft kann diese Frage auch gar nicht eindeutig beantwortet werden (man denke etwa an die glückliche, befriedete, aber unfreie Gesellschaft in Aldous Huxleys Brave New World). Grundsätzlich entwirft das utopisch/dystopische Schreiben Versionen anderer Welten, von Welten also, die nicht mit der realen Welt übereinstimmen, da sie entweder in der Zukunft liegen oder an unbekannten Orten angesiedelt sind. Dieses Postulat anderer Welten ist bei Utopie und Dystopie darüber hinaus stets mit normativen oder politischen Implikationen verbunden: Nicht-normative Utopien oder Dystopien gibt es nicht. Im Falle, dass Utopien oder Dystopien ihre Verbindung mit der Sphäre des realweltlich Politischen verlieren, gehen sie damit zugleich ihres Status als Utopien oder Dystopien verlustig. Sie kippen dann gewissermaßen in den Genrebereich der Science-Fiction oder Fantasy. Die Kombination einer tendenziell nicht-realistischen Erzählwelt mit einer gleichzeitigen interpretatorischen Anbindung an politische Konkreta wird in vielen utopischen und dystopischen Texten durch einen verstärkten Rückgriff auf kontrafaktische Referenzstrukturen geleistet.

Dokufiktion: Die konstitutive Faktenbindung des Dokumentarismus scheint eine Einbindung kontrafaktischer Elemente zunächst auszuschließen. Für gewöhnlich versuchen dokumentarische Texte gerade nicht, von realweltlichen Fakten (kontrafaktisch) abzuweichen, sondern erheben vielmehr den Anspruch, diese realweltlichen Fakten einigermaßen adäquat - also im Modus der Faktik zu reproduzieren. ${ }^{434}$ Eine Genrevariante des künstlerischen Dokumentarismus bilden allerdings Formen ,kreativer‘ Dokumentarismen oder sogenannte Dokufiktionen - Begriffsbildungen, die vordergründig paradox anmuten, deren interpretatorisches Potenzial aber just aus dieser Paradoxie erwächst. So können etwa sogenannte Mockumentarys, also fiktionale Pseudo-Dokumentationen, auf kontrafaktische Referenzstrukturen zurückgreifen, um in einem parodistischen oder satirischen Modus Medienkritik zu betreiben. Auch im politischen Kabarett ist die

433 Vgl. Zipfel: Fiktion, Fiktivität, Fiktionalität, S. $101 \mathrm{f}$.

434 Eines der Grundprobleme des Dokumentarismus, dass nämlich eine dokumentarische Faktenreproduktion die Fakten niemals vollkommen unverfälscht und unverkürzt darstellen kann, ist dabei zunächst ohne Belang: Wichtig ist allein die Tatsache, dass ein Dokumentarismus, der gänzlich auf eine Entsprechung zwischen Material und Darstellung verzichten würde, gar kein Dokumentarismus mehr wäre. 
kontrafaktische Überzeichnung oder Verballhornung dokumentarischen Faktenmaterials weit verbreitet. Die satirisch-kabarettistische Überzeichnung einer Politikeraussage etwa lässt sich, je nach Ausmaß und Form der Realitätsabweichung, durchaus als kontrafaktische Faktenvariation begreifen. Im postmodern-selbstreflexiven Dokumentarismus von Elfriede Jelinek oder Kathrin Röggla schließlich werden dokumentarische Formate selbst zum Material eines kreativen, kontrafaktischen Dokumentarismus oder Meta-Dokumentarismus. Dokumentarismus wird hier nicht als eine Erzählform mit gesteigertem Informationswert zum Zwecke der Aufklärung eingesetzt. Stattdessen werden kontrafaktische Erzählverfahren auf bereits bestehende dokumentarische Diskurse angewandt, um sie auf diese Weise künstlerisch zu verfremden und damit (politisch) zu kommentieren.

Autofiktion: Sogenannte Autofiktionen, also fiktionalisierte oder semi-literarische Formen der Autobiografie, sind angesichts ihres konstitutiven Bezugs auf realweltliche Fakten - die Lebensdaten einer realen Person - prinzipiell fähig, kontrafaktische Elemente zu integrieren. Die transfiktionale Doppelreferenzstruktur der Kontrafaktik - also die Verbindung von indirekter Referenz auf einen realweltlichen Sachverhalt mit einer Referenz auf die binnenfiktionale Variation desselben Sachverhalts - lässt sich gut an das definierende Charakteristikum der Autofiktion anschließen, nämlich „die Verbindung von zwei sich eigentlich gegenseitig ausschließenden Praktiken: die referentielle Praxis und die FiktionsPraxis. “ ${ }^{435}$ Bedingung für eine mögliche Aktualisierung des genuin kontrafaktischen Potenzials der Autofiktion durch eine größere Leserschaft ist allerdings, dass die biografischen Sachverhalte, die innerhalb der Autofiktion variiert werden, auch unabhängig von der Autofiktion bekannt oder zumindest leicht rekonstruierbar sind. Leicht lässt sich das kontrafaktische Potential etwa bei der Variation der Biografien berühmter Persönlichkeiten erschließen (wobei hier die Übergänge zum Genre der Alternativgeschichte fließend sind). ${ }^{436} \mathrm{Au}$ tofiktionen von weniger bekannten Autoren dürften hingegen eher selten als kontrafaktische Texte wahrgenommen werden (es sei denn, es würden sich hier kontrafaktische und fantastische Weltvergleichsverhältnisse überlagern). Ebenfalls wenig kontrafaktik-affin sind Varianten formal-selbstreflexiven, autofiktionalen Erzählens, die nicht auf erkennbare Weise faktische und kontrafaktische Aspekte der Personendarstellung miteinander kombinieren, sondern eher allgemein epistemologische oder erinnerungstheoretische Fragen verhandeln.

435 Frank Zipfel: Autofiktion. Zwischen den Grenzen von Faktualität, Fiktionalität und Literarität? In: Simone Winko / Fotis Jannidis / Gerhard Lauer (Hg.): Grenzen der Literatur. Zu Begriff und Phänomen des Literarischen. Berlin 2009, S. 285-314, hier S. 311.

436 Siehe grundlegend zur Autofiktion Martina Wagner-Egelhaaf (Hg.): Auto(r)fiktion. Literarische Verfahren der Selbstkonstruktion. Bielefeld 2013. 
Schlüsselroman: Als besonders ergiebig erweist sich die Frage nach kontrafaktischen Referenzstrukturen im Falle von Schlüsselromanen, bei Romanen also, die, wie Johannes Franzen schreibt, „Personen der Alltagswirklichkeit wiedererkennbar als Figuren in ihre vordergründig fiktionale Handlung integrieren. “437 Bereits die Begriffe „Alltagswirklichkeit“, „wiedererkennbar“ und „vordergründig fiktional“ lassen dabei gewisse Überschneidungen mit dem Vokabular der in dieser Studie vorgestellten Fiktionstheorie der Kontrafaktik erkennen. Auch beim Schlüsselroman handelt es sich um ein Erzählphänomen, das sich nicht allein durch manifeste Eigenschaften an der Textoberfläche charakterisieren lässt, sondern das wesentlich von einer bestimmten Form der Rezeption abhängt: Die fiktionale Welt des Schlüsselromans kann - wie alle fiktionalen Welten - nicht aus sich selbst heraus garantieren, dass ein konkreter Faktenbezug tatsächlich hergestellt wird (es sei denn, es würden nicht-fiktionale Paratexte beigefügt, die dann aber - ebenso wie bei der Kontrafaktik - nicht mehr eigentlich zum fiktionalen Text hinzuzählen würden ${ }^{438}$ ). Indem man einen Roman als ,Schlüsselroman' bezeichnet, gibt man allerdings bereits zu erkennen, dass man eine Beziehung zwischen fiktionaler Person und realer Figur erkannt und für deutungsrelevant befunden hat. Die Folgefrage kann dann nur noch lauten, ob man es hier mit einer faktischen oder mit einer kontrafaktischen Personendarstellung zu tun hat (Realistik und Fantastik scheiden als Weltvergleichsverhältnisse tendenziell aus, da den Eigenschaften konkreter Personen, die eine Identifikation dieser Personen erlauben, immer der Status von Einzelinformationen und nicht nur von allgemeinen Realitätsannahmen zukommt ${ }^{439}$ ). Die Bewertung dieser Frage wird nicht zuletzt davon abhängen, welche normativen Wertungen man mit der jeweiligen Darstellung verbindet: Eine Person, die sich in einem Roman verleumdet

437 Johannes Franzen: Indiskrete Fiktionen. Schlüsselromanskandale und die Rolle des Autors. In: Andrea Bartl / Martin Kraus (Hg.): Skandalautoren. Zu repräsentativen Mustern literarischer Provokation und Aufsehen erregender Autorinszenierung. Bd. 1. Würzburg 2014, S. 67-92, hier S. $77 \mathrm{f}$.

438 Solche faktual-paratextuellen Hinweise werden im Falle von Schlüsselromanen - zumindest bei ihrer Erstveröffentlichung - so gut wie niemals von den Autoren selbst platziert, da die Autoren von Schlüsselromanen ja für gewöhnlich gerade nicht zugeben wollen, dass sie einen Schlüsselroman verfasst haben, unter anderem deswegen, weil bei der verleumdenden Darstellung realer Personen juristische Konsequenzen drohen. Man denke an den Fall von Maxim Billers Roman Esra. Vgl. Uwe Wittstock: Der Fall Esra. Ein Roman vor Gericht. Über die neuen Grenzen der Literaturfreiheit. Köln 2011.

439 Nicht prinzipiell auszuschließen ist allerdings eine Überlagerung von Fantastik und Kontrafaktik; fantastische Schlüsselromane wären also prinzipiell vorstellbar. Siehe hierzu die Diskussion zum Status von Tolkiens Sauron/Herr der Ringe-Figur in Kapitel 5.1. Realistik, Fantastik, Kontrafaktik, Faktik. 
sieht und die diesen Roman entsprechend als ,Schlüsselroman“ anprangert, bringt damit bereits implizit zum Ausdruck, dass sie eine signifikante Diskrepanz zwischen ihrer eigenen, realweltlichen Faktizität und der fiktionalen Figurendarstellung zu erkennen glaubt, dass es sich ihrer Ansicht nach hier also um eine ,kontrafaktische‘ Figurendarstellung handelt (eine Unterstellung, welche die Autoren der jeweiligen Texte selbstverständlich meistens abstreiten ${ }^{440}$ ). Allerdings sind Schlüsselromane nicht grundsätzlich kontrafaktisch: Eine nicht-kontrafaktische Variante des Schlüsselromans liegt vor in Fällen, bei denen nicht die realitätsvariierende Darstellung einer bestimmten Person als skandalös empfunden wird, sondern vielmehr der Umstand, dass bestimmte sensible Informationen überhaupt - und sei es unter dem Vorwand der literarischen Fiktion publik gemacht werden. Problematisch wäre hier nicht so sehr die (verleumderische) Faktenvariation als vielmehr die schlichte Indiskretion.

Satire: Während das Genre des Schlüsselromans prinzipiell sowohl mit faktischen als auch mit kontrafaktischen Realitätsreferenzen kompatibel ist, handelt es sich bei der fiktionalen Satire um ein Erzählverfahren, das notwendigerweise eine kontrafaktische Referenzstruktur aufweist (allerdings nur unter der Voraussetzung, dass die Satire sich auf die reale Welt und nicht auf andere fiktionale Texte bezieht: Bei Literatursatiren handelt es sich nicht um einen Fall von Kontrafaktik, sondern um einen Fall von Intertextualität). ${ }^{441}$ Die fiktionale Satire - ein Text also, der nicht in einem isolierten satirischen Sprechakt besteht, sondern eine eigene fiktionale Welt entwirft ${ }^{442}$ - ist gerade

440 Für gewöhnlich findet beim Schlüsselroman der ,initiale[...] Akt der Entschlüsselung“ in den Medien statt, so etwa im Falle des Skandals um Martin Walsers Roman Tod eines Kritikers, der durch einen offenen Brief des FAZ-Herausgebers Frank Schirrmacher ausgelöst wurde, in dem dieser Walser vorwarf, mit der Figur André Erl-König ein antisemitisches Portrait Marcel Reich-Ranickis gezeichnet zu haben (Franzen: Indiskrete Fiktionen. Schlüsselromanskandale und die Rolle des Autors, S. 84).

441 Albrecht etwa charakterisiert das Verfahren kontrafaktischer Imaginationen, die in ,ironischer Absicht“ eine bestimmte Position „karikaturistisch überzeichne[n]“ als „satirische Mimesis“ (Andrea Albrecht: Kontrafaktische Imaginationen zum Logischen Empirismus. Max Horkheimer und Ludwik Fleck. In: Scientia Poetica 20 (2016), S. 364-394, hier S. 386 - Hervorhebung M. N.).

442 Bei einem isolierten Einsatz satirischen Sprechens würde es sich nicht um Kontrafaktik, sondern um einen Fall uneigentlicher Rede handeln. Eine isolierte satirische Aussage verhält sich zur Satire als fiktionale Gattung in etwa so wie die Metapher zur Gattung der Allegorie. Vgl. Urs Meyer: Metapher - Allegorie - Symbol. In: Thomas Anz (Hg.): Handbuch Literaturwissenschaft. Bd. 1: Gegenstände und Grundbegriffe. Stuttgart 2007, S. 105-110, hier S. 105. 
dadurch definiert, dass sie realweltliches Faktenmaterial in kritisch-humoristischer Weise im Rahmen ihrer fiktionalen Welt variiert. ${ }^{443}$

Angesichts der hohen Affinität der genannten Genres zu kontrafaktischen Referenzstrukturen überrascht es nicht, dass diese Genres untereinander häufig Hybridbildungen eingehen. So wird gerade in Schlüsselromanen nicht selten auf satirische Erzählverfahren zurückgegriffen (man denke etwa an Martin Walsers Darstellung von Marcel Reich-Ranicki im Roman Tod eines Kritikers); Manifestationsformen des kreativen Dokumentarismus enthalten oftmals autofiktionale Elemente (bei der Diskussion von Rögglas wir schlafen nicht wird darauf zurückzukommen sein); und Alternate History, Utopie und Dystopie weisen hinsichtlich ihrer Genreentwicklung, ihrer zentralen Themen sowie ihrer Leserschaft eine bedeutende Schnittmenge auf.

\subsection{Metafaktizität}

Ein spezifisches Verhältnis von Fakten und Faktenabweichung ist für die Kontrafaktik als Erzählphänomen konstitutiv. Zahlreiche kontrafaktische Texte problematisieren das Verhältnis von Fakt und Erfindung jedoch nicht nur über ihren spezifischen Fiktionsstatus als kontrafaktische Werke, sondern auch auf der Ebene ihres narrativen Inhalts. In der Forschung ist mehrfach darauf hingewiesen worden, dass eine erstaunlich große Anzahl kontrafaktischer Texte selbst Fragen der Konstitution von Fakten, von Faktenmanipulation und von Täuschung thematisiert. ${ }^{444}$ Diese Formen der Faktendiskussion innerhalb kontrafaktischer Werke sollen im Folgenden als ,metafaktisch“, das entsprechende Erzählphänomen als ,Metafaktizität' bezeichnet werden. ${ }^{445}$

Um das mögliche Spektrum derartiger metafaktischer Reflexionen innerhalb kontrafaktischer Texte anzudeuten, kann auf einige der Referenzbeispiele zurückgegriffen werden: Als der ,Jew Hunter` Hans Landa in Tarantinos Inglourious Basterds erkennen muss, dass ein Sieg der Deutschen nicht mehr möglich ist und er selbst somit Gefahr läuft, sich nach Ende des Krieges vor einem jüdischen Tribunal für seine Taten verantworten zu müssen, entschließt er sich zu einem Deal

443 Siehe zur notwendigen Referenz der Satire auch Gertrud Maria Rösch: Clavis Scientiae. Studien zum Verhältnis von Faktizität und Fiktionalität am Fall der Schlüsselliteratur. Tübingen 2004, S. 79-83; Blume: Fiktion und Weltwissen, S. 207.

444 Vgl. Helbig: Der parahistorische Roman, S. 90 f.; Hellekson: The Alternate History, S. 30 f. 445 Siehe hierzu meine Ausführungen in Navratil: Lying in Counterfactual Fiction. 
mit den ,Basterds‘ und der englischen Regierung: Im Gegenzug für die Ermordung Hitlers und der NS-Führung fordert er - nebst einer beträchtlichen materiellen Rekompensation -, dass seine Verbrechen im Dienste der Nazis öffentlich als bloße Tarnaktionen ausgegeben werden und er selbst in den Rang eines Kriegsheld erhoben wird, der einen unverzichtbaren Beitrag zur Beendigung des Zweiten Weltkriegs geleistet habe. Mit der lakonischen Frage „What shall the history books read?“ stellt Landa den Anführer der Basterds vor die Wahl, die skandalöse Geschichtslüge zu unterstützen und einen Hauptverantwortlichen der nationalsozialistischen Judenhetze und -vernichtung zu decken, damit aber zugleich den Krieg zu beenden - oder aber auf der Wahrheit zu beharren, auf die Gefahr hin, dass der Krieg Millionen weitere Opfer fordern könnte. Tarantinos Film verhandelt damit auf der inhaltlichen Ebene die Probleme der Geschichtsfälschung, der opportunistischen Realpolitik und des Widerstreits zwischen Utilitarismus und einer Prinzipienethik, die metonymisch mit dem totalen Signifikanten ,Auschwitz' in Verbindung steht. Wenig überraschend wird die Option einer Geschichtslüge bezüglich des Holocaust in Inglourious Basterds entschieden abgelehnt.

Weniger augenfällig, dafür aber kontinuierlich über den gesamten Roman verteilt, wird das Thema der Verfügbarkeit von Fakten in Michel Houellebecqs Unterwerfung verhandelt. Weite Strecken des Romans bestehen aus Gesprächen des Protagonisten François mit Personen, die anscheinend über sehr viel umfassendere Informationen zur politischen Lage innerhalb des fiktiven Zukunftsfrankreich verfügen als François selbst. Die erstaunliche - und nur bedingt glaubwürdige Ahnungslosigkeit des Protagonisten bezüglich politischer Fragen liefert in Unterwerfung den erzählpragmatischen Vorwand, um die gesellschaftliche Situation und vor allem die Motivationen der islamischen Partei ausführlich zu erläutern. Darüber hinaus erfüllt die Uninformiertheit des Protagonisten eine metafaktische Funktion: Der Status relevanter politischer Informationen als Geheimwissen beziehungsweise als Wissen, das nur einer kleinen Gruppe von Personen zugänglich ist, lässt sich auf die reale rechtspopulistische Angst vor einer islamistischen Verschwörung rückbeziehen, auf jene Angst also, die gerade den - oder doch einen wichtigen - Ausgangspunkt von Houellebecqs kontrafaktisch-dystopischer Zukunftsvision bildet. Ebenfalls als metafaktisches Element lässt sich die den Roman durchziehende Thematik der Manipulation medialer NachrichtenBerichterstattung deuten, welche den rechtspopulistischen Vorwurf der ,Lügenpresse' kontrafaktisch ausgestaltet.

Das wohl bekannteste und meistdiskutierte Beispiel der Metafaktizität innerhalb der kontrafaktischen Literatur - neben den systematischen Geschichtsklitte- 
rungen des Ministry of Truth in George Orwells $1984^{446}$ - findet sich in Philip K. Dicks Roman The Man in the High Castle. In der Erzählwelt von Dicks Roman wird verschiedentlich ein fiktives Werk mit dem Titel The Grasshopper Lies Heavy erwähnt, verfasst von einem Autor namens Hawthorne Abendsen, auch ,das Orakel vom Berge' genannt. Während in der Welt von Dicks Roman die Nationalsozialisten den Krieg gewonnen haben und das Gebiet der USA zwischen dem Dritten Reich und dem japanischen Kaiserreich aufgeteilt wurde, erzählt Abendsens Werk The Grasshopper Lies Heavy vom Sieg der Achsenmächte, von der Gefangennahme Hitlers und Goebbels' und vom Aufstieg des britischen Empire zur mächtigsten Nation der Welt.

In Abbildung 3 wird diese mehrfache ,Faktenschachtelung“ in The Man in the High Castle schematisch dargestellt. Während es in der realen Welt unzweifelhafter Fakt ist - dargestellt durch ein großes F -, dass Nazi-Deutschland den Krieg verloren hat, haben die Nazis in Dicks Roman den Krieg gewonnen. In Abendsens Text hingegen unterliegen die Nazis im Krieg, was die Geschichtsversion seines Buches wiederum näher an die Welt der realweltlichen Leser heranrückt. ${ }^{447}$

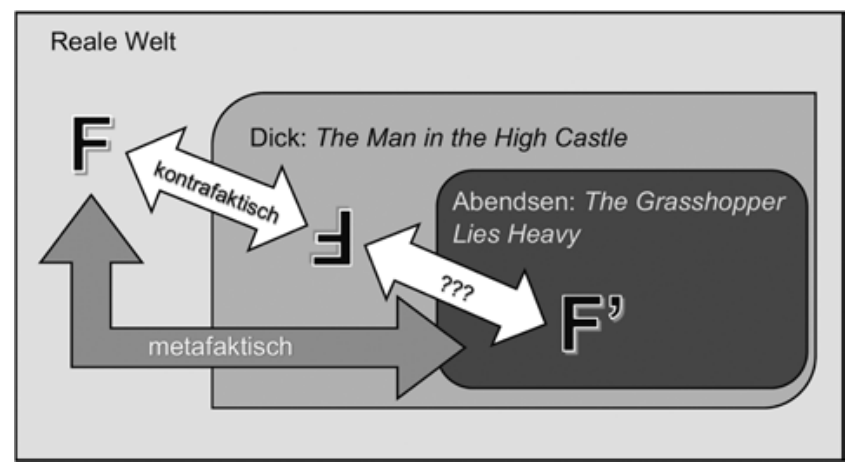

Abbildung 3: Metafaktizität in Philip K. Dicks The Man in the High Castle.

4461984 wurde meines Wissens bisher nicht als Beispiel kontrafaktischer Literatur diskutiert. Mit Blick auf den stalinistischen Terror, den Orwell in seinem Roman implizit kommentiert, ließen sich kontrafaktische Lesarten des Romans allerdings leicht plausibilisieren. Die kontinuierlichen Geschichtsumschreibungen des Ministry of Truth in Orwells Roman etwa können auf die Geschichtsklitterungen des stalinistischen Regimes bezogen werden.

447 Françoise Lavocat beschreibt diese Struktur wie folgt: „M (die reale Welt) schließt M1 ein (die Welt des Romans von Philip K. Dick), und diese schließt ihrerseits M2 ein (den Roman im Roman mit dem Titel Schwer liegt die Heuschrecke, der eine weitaus größere Nähe zu M als zu M1 aufweist.“ (Françoise Lavocat: Kontrafaktische Narrative in Geschichte und Fiktion. In: Johannes Franzen u. a. (Hg.): Geschichte der Fiktionalität. Diachrone Perspektiven auf ein kulturelles Konzept. Baden-Baden 2018, S. 253-267, hier S. 262). 
Jörg Helbig bemerkt zum Buch im Buch innerhalb von Dicks Roman: „Ein einfacher Analogieschluß führt den Leser zu der Erkenntnis, das The Grasshopper Lies Heavy zu The Man in the High Castle im gleichen Bezugsverhältnis steht wie The Man in the High Castle zur empirischen Realität, der fiktive Autor Abendsen zum realen Autor Dick die gleiche Position bezieht, wie Dick zur Wirklichkeit.“448 Dieser ,einfache Analogieschluss` erweist sich bei genauerer Betrachtung allerdings als allzu simpel, ja reduktiv. Während nämlich die von Dick entworfene Welt ganz offensichtlich vom enzyklopädischen Wissen über den Zweiten Weltkrieg abweicht und somit von der Warte des realweltlichen Modell-Lesers aus zweifellos als kontrafaktisch zu klassifizieren ist, bleibt der Status der von Abendsen geschilderten Welt innerhalb von Dicks Roman unklar beziehungsweise wird von unterschiedlichen Figuren unterschiedlich bewertet: Während einige Figuren Abendsens Werk als Alternate History-Erzählung - also als fiktionales Fantasieprodukt betrachten, offenbart sich für die Protagonistin Juliana Frink am Ende des Romans die ,tiefere Wahrheit‘ des Textes: Dies geschieht, als sie erfährt, dass Abendsen beim Verfassen des Buches das auch im sonstigen Roman allenthalben befragte Orakelbuch I Ging zu Rate gezogen hat, sodass es sich bei The Grasshopper Lies Heavy in gewissem Sinne um einen faktualen Text zu handeln scheint. Von der nationalsozialistischen Zensur wiederum wird Abendsens Buch verboten, wobei erneut unklar bleibt, ob hier lediglich eine fiktionale Erzählung zensiert wird, die das triumphale Siegernarrativ der Nazis literarisch unterminiert, oder ob nicht vielmehr faktuales Geheimwissen über den realen Kriegsverlauf unterdrückt werden soll. Der pragmatische Status des metafaktischen Textes The Grasshopper Lies Heavy wird in Dicks Roman somit auf reizvolle Weise in der Schwebe gehalten zwischen kontrafaktisch-fiktionalem Werk, Verschwörungstheorie und faktualer Prophetie. ${ }^{449}$ Auch mit Blick auf seine metafaktischen Reflexionen erweist sich The Man in the High Castle somit als legitimer Klassiker der kontrafaktischen Geschichtsimagination, der in seiner Komplexität und interpretatorisch produktiven Ambivalenz bereits weit über die Mehrzahl der nachfolgenden Genrebeispiele hinausgeht.

In den meisten kontrafaktischen Werken beziehen sich metafaktische Diskussionen allerdings weder auf ,kontra-kontrafaktische Geschichtsverläufe“450 (wie sie ja auch bei Dick nur eingeschränkt vorliegen) noch auch auf Metakommentare zum Konstruktionscharakter literarischer oder historiografischer Rede. Metafaktische Elemente in kontrafaktischen Texten sind selbst meist weder kontrafaktisch

448 Helbig: Der parahistorische Roman, S. 172.

449 Vgl. Dannenberg: Coincidence and Counterfactuality, S. 208-210.

450 Helbig: Der parahistorische Roman, S. 90. 
noch auch metafiktional, sondern eher non- oder postfaktisch, insofern sie sich auf die Produktion unwahrer Aussagen mit Täuschungsabsicht beziehen: Die Geschichtsrevisionen und die Propaganda des Ministry of Truth in Orwells 1984, Hans Landas selbstnobilitierende Geschichtsfälschung in Tarantinos Inglourious Basterds, die Vertuschung des Genozids an den Juden in Harris' Fatherland und die Medienmanipulationen in Houellebecqs Unterwerfung - all diese Formen der Faktenmanipulation haben mit Kontra-Kontrafaktik oder Metafiktionalität weniger zu tun als mit handfesten Lügen. Problematisiert wird anhand dieser metafaktischen Elemente nicht der Status des Kontrafaktischen innerhalb kontrafaktischer Werke oder das Zustandekommen fiktionaler Aussagen; problematisiert werden vielmehr die Entstehungsbedingungen und vor allem die Wirkungen faktualer Fiktivität. Während also ein realweltlich unwahrer Sachverhalt innerhalb der fiktionalen Welt unter bestimmten Bedingungen dazu führen kann, dass ein Text der Kontrafaktik zugeschlagen wird, betrifft die metafaktische Verhandlung diegetisch unwahrer Propositionen innerhalb kontrafaktischer Texte selbst meist eher - wenn auch nicht immer Fragen der Täuschung, Faktenmanipulation oder Lüge.

Auf scheinbar paradoxe Weise eignen sich somit gerade metafaktische Elemente als künstlerische Mittel einer Kritik an Fake News und verwandten Phänomenen: Just der epistemisch komplexe Status metafaktischer Elemente als gleichsam kategorial disparate, geschachtelte Unwahrheit - nämlich unwahre Aussagen mit Täuschungsabsicht (Lügen etc.) innerhalb von offenkundig realitätsvariierenden fiktionalen Welten (Kontrafaktik) - kann dazu beitragen, die Aufmerksamkeit zu schärfen für die oftmals entschieden negativen Effekte von Täuschung, Faktenmanipulation und Lüge innerhalb der jeweiligen Erzählwelten. Tatsächlich scheint die künstlerische Intention des Einsatzes metafaktischer Elemente häufig in der Verteidigung objektiver Wahrheit respektive einer Kritik an (politischen) Unwahrheiten und Fake News zu bestehen. ${ }^{451}$

Trotz einer gewissen Dominanz von Ausprägungen der Metafaktizität, die innerhalb ihrer jeweiligen fiktionalen Welt fiktive Aussageinhalte mit Täuschungsabsichten verbinden, lässt sich der genaue Fiktionsstatus metafaktischer Elemente nicht verallgemeinernd, sondern nur fallweise angeben (das oben diskutierte Beispiel aus Dicks The Man in the High Castle stellt ja gerade einen besonders komplexen, wenig eindeutigen Fall von Metafaktizität dar). Auf welche genaue Weise sich metafaktische Elemente in einzelnen kontrafaktischen Texten zu Fragen der Fiktionalität, Fiktivität und Literarizität verhalten, kann dabei von einiger Bedeutung sein für die Beantwortung der Frage, ob man

451 Vgl. Navratil: Lying in Counterfactual Fiction. 
einen kontrafaktischen Text eher der Gruppe des traditionellen, gleichsam referenzoptimistischen Romans oder aber derjenigen des selbstreflexiv-postmodernen Romans zuschlagen wird. Metafaktizität, die sich mit den vergleichsweise simplen Fragen von Täuschung und Lüge befasst, impliziert nicht notwendig ein kritisches Bewusstsein bezüglich der Zeichenhaftigkeit oder der Referenzproblematik (literarischer) Texte; Fragen von Täuschung und Lüge werden schließlich auch in den einfachsten Detektiv-Geschichten verhandelt. Metafaktische Aussagen und Textelemente hingegen, die Fragen der Literarizität, der Sprachlichkeit von Wahrheitsaussagen oder sogar des kontrafaktischen Erzählens selbst aufwerfen, rücken einen literarischen Text tendenziell in die Nähe selbstreflexiv-postmoderner Prosa, oder, im speziellen Fall des historischen Erzählens, in die Nähe dessen, was Linda Hutchon als ,historiographic metafiction' bezeichnet hat. ${ }^{452}$ Weder die bloße Existenz eines kontrafaktischen Textes noch auch das Vorliegen metafaktischer Elemente innerhalb dieses Textes erlaubt also an und für sich bereits eine Entscheidung darüber, ob man es hier mit einem eher konventionell-referenzoptimistischen oder mit einem referenzsubversiven, metareflexiven oder postmodernen Text zu tun hat. Welche der beiden Positionen respektive welches Mischverhältnis zwischen ihnen jeweils vorliegt, wird sich letztlich immer nur anhand des einzelnen Textes entscheiden lassen.

452 Zur Einordnung kontrafaktischer historischer Romane innerhalb des Genres des historischen Romans siehe Kapitel 10. Historisches Erzählen als Kontrafaktik. 